\title{
A Study of the Interaction between Typhoon Francisco (2013) and a Cold-Core Eddy. Part II: Boundary Layer Structures ${ }^{\mathfrak{O}}$
}

\author{
Zhanhong Ma, Jianfang Fei, Xiaogang Huang, Xiaoping Cheng, and Lei Liu \\ College of Meteorology and Oceanography, National University of Defense Technology, Nanjing, China
}

(Manuscript received 11 December 2019, in final form 12 June 2020)

\begin{abstract}
In Part II of this study, the influence of an oceanic cold-core eddy on the atmospheric boundary layer structures of Typhoon Francisco (2013) is investigated, as well as a comparison with the cold wake effect. Results show that the eddy induces shallower mixed-layer depth and forms stable boundary layer above and near it. The changes of these features shift from northwest to southeast across the storm eye, following the translation of Francisco over the eddy. Nonetheless, the decrease in mixed-layer depth and formation of stable boundary layer caused by the cold wake are located at right rear of the storm. The sensible heat fluxes at the lowest atmospheric model level are mostly downward over the sea surface cooling region. Due to their different relative locations with Francisco, the diabatic heating in the northwest quadrant of the storm can be more effectively inhibited by the cold-core eddy than by the cold wake. The asymmetric characteristics of surface tangential wind are less sensitive to sea surface cooling than those of surface radial wind, implying a change in surface inflow angle. Different from previous studies, the surface inflow angle is found to be reduced especially above the cold-core eddy and cold wake region. An analysis of radial wind tendency budget indicates that the decrease in radial pressure gradient is dominant in changing the acceleration rate of surface radial wind, rather than the decrease in the Coriolis and centrifugal forces, and therefore more outward surface flow is induced by both the cold-core eddy and cold wake.
\end{abstract}

\section{Introduction}

This is the second part of a study investigating the interaction of a tropical cyclone (TC) with an oceanic cold-core eddy. Ma (2020, hereafter Part I) recorded a unusually rapid weakening event of Typhoon Francisco (2013) after crossing over a cold-core eddy, which was intensified by two prior typhoons. During the typhooneddy interaction, dramatic ocean response resulted in halved supply of surface enthalpy flux and diminished latent heat release associated with suppressed convective bursts. This part of the study will extend the work of Part I to the influence of oceanic feedback on TC boundary layer characteristics.

The atmospheric boundary layer (including the surface layer) is the lowest part of a TC that determines how the air-sea exchange of heat, moisture, and momentum is

\footnotetext{
Supplemental information related to this paper is available at the Journals Online website: https://doi.org/10.1175/JAS-D-190339.s1.
}

Corresponding author: Zhanhong Ma, mazhanhong17@nudt.edu.cn accomplished within the TC system. Since it is directly affected by the underlying surface and is characterized by strong turbulence, the TC boundary layer exhibits unique properties relative to the other part of the TC, such as the breakdown of thermal stability and gradient wind balance (Kepert 2010). Despite the importance of boundary layer processes, many features of the TC boundary layer are still not well understood. For instance, there has been no consensus on the practical definition of boundary layer height. One of the commonly used definitions can be referred to as the thermodynamic boundary layer depth (Anthes and Chang 1978), which is defined as the level where virtual potential temperature $\left(\theta_{v}\right)$ is $0.5 \mathrm{~K}$ larger than the surface value. This definition is referred to as HVTH in this study. In essence it assumes that the boundary layer is a well-mixed layer with nearly constant potential temperature. However, the boundary layer is not well mixed in the upper part, and is usually much deeper than the mixed layer (Kepert et al. 2016). The other commonly used definition regards the boundary layer as the friction-forced inflow layer (Smith et al. 2009). This definition is advocated by recent observational 
and theoretical studies (e.g., Zhang et al. 2011; Kepert et al. 2016). However, this dynamic definition has its own limitations, since the friction-induced inflow cannot be easily isolated, and there may be no inflow layer in some regions of TCs. There are also some other definitions, such as regarding the boundary layer as an Ekman-like layer that is proportional to the square root of turbulent diffusivity and inversely proportional to the square root of inertial stability (Kepert 2001), using the height of maximum wind speed (Bryan and Rotunno 2009), or simply adopting a constant height layer (Ma et al. 2013a). This study will compare the former two commonly used definitions.

The changes in sea surface state caused by wind forcing of TCs will in turn give feedback to the boundary layer characteristics of TCs. Lee and Chen (2012) examined the symmetric and asymmetric features of boundary layer height using a fully coupled numerical model. They found an asymmetric decrease of HVTH in the rear-right quadrant due to the cold wake; they also cautioned the validity of using inflow layer to define the dynamic boundary layer depth as well as the representativeness of azimuthally averaged boundary layer properties in Zhang et al. (2011). This latter viewpoint is nonetheless critiqued by Zhang et al. (2014) primarily for their calculation of dynamic boundary layer and misunderstanding of Zhang et al. (2011). Wu et al. (2016) also found shallower mixed-layer depth over the cold wake, with the depth decrease being more pronounced as the sea surface temperature (SST) decrease is larger.

The boundary layer of a TC is typically characterized by convective instability. However, there are extreme cases where the sea surface tends to be cooler than the surface layer. Black and Holland (1995) recorded a stable boundary layer when air flowed over the cold wake region, and they found that the sensible heat fluxes are downward into the ocean in that region. The downward transport of sensible heat should be associated with the stability nature of TC boundary layer as illustrated in Ma et al. (2015). Lee and Chen (2014) conducted further work and proposed a positive contribution of cold wake-induced stable boundary layer, in that the air in the stable boundary layer stayed longer due to suppressed convection, thereby possessing higher energy, and the inflow was stronger due to increased inflow angle. These findings are advocated by $\mathrm{Wu}$ et al. (2016) based on the case simulation of Typhoon Megi (2010). Lee and Chen (2014) speculated that the enhanced inflow angle is attributed to stronger gradient wind imbalance, since the reduced turbulent mixing would cause decreased wind speed in the stable boundary layer. Nonetheless, they did not provide evidence for such deductions.
Relative to the progress in understanding the cold wake effect on TC boundary layer, there has been very limited literature focusing on the role of mesoscale oceanic eddies, including both warm-core eddy and cold-core eddy. Current studies of TC-eddy interactions have paid very much attention on the intensity changes than on the boundary layer characteristics (e.g., Hong et al. 2000; Shay et al. 2000; Lin et al. 2005; Ma et al. 2013b; Walker et al. 2014). In Part II of this study, the contribution of cold-core eddy to symmetric and asymmetric changes of boundary layer structures will be examined, using the model output in Part I. The tracking of TC centers is based on the default automatic moving nest in the Weather Research and Forecasting (WRF) Model. Results based on the location of minimum sea level pressure gives consistent conclusions with quantitative differences (not shown). Following Part I, the effect of cold-core eddy is isolated by the difference between the control run (COUP) and the eddy-removed run (NOED), namely, the contribution of enhanced sea surface cooling due to the presence of cold-core eddy, while the cold wake effect is diagnosed by the difference between NOED and the uncoupled run (UNCP). The primary results are presented in section 2 , and a summary is provided in section 3 .

\section{Results}

\section{a. Storm size}

The time evolution of radii of azimuthally averaged maximum surface wind (RMW) and hurricane-force wind $\left(33 \mathrm{~m} \mathrm{~s}^{-1}\right.$; R33) is shown in Fig. 1. The eyewall contraction is closely related to the storm intensification (Shapiro and Willoughby 1982; Willoughby et al. 1982), while they generally do not cease simultaneously (Kieu 2012; Stern et al. 2015). The RMW in all three experiments keeps invariant initially when the storms still intensify slightly (Fig. 9 in Part I), and then it is enlarged with time accompanying subsequent storm weakening. NOED shows smaller RMW than COUP approximately during the eddy interaction, and their difference diminishes gradually after the departure of Francisco from the eddy. The UNCP experiment gives the smallest RMW, with its difference from NOED being apparent until the end of the simulation. Overall, the discrepancies of RMW among three experiments are consistent with those of intensities (Fig. 2).

Both of the cold-core eddy and cold wake lead to decreased radial pressure gradient (Fig. 2), and therefore reduced radial inflow (not shown), which is largely responsible for the contraction or enlargement of RMW (Kieu 2012). At the early stage of the simulation, the 
(a) RMW (km)

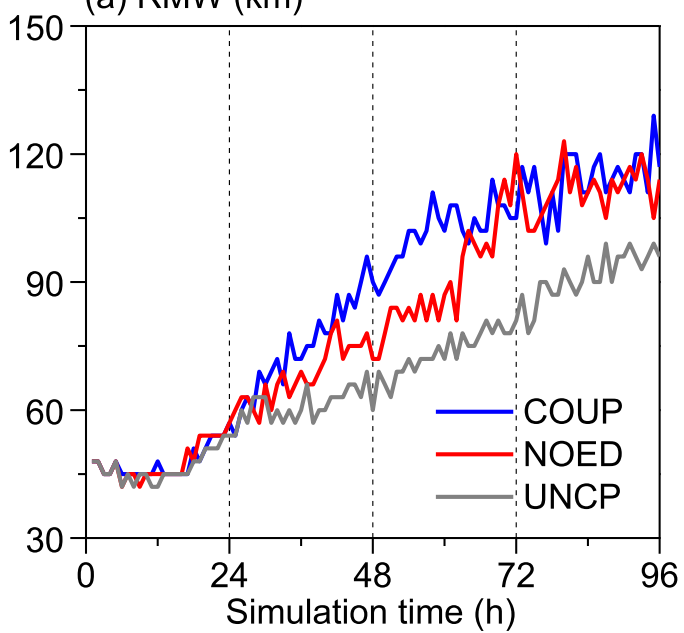

(b) R33 (km)

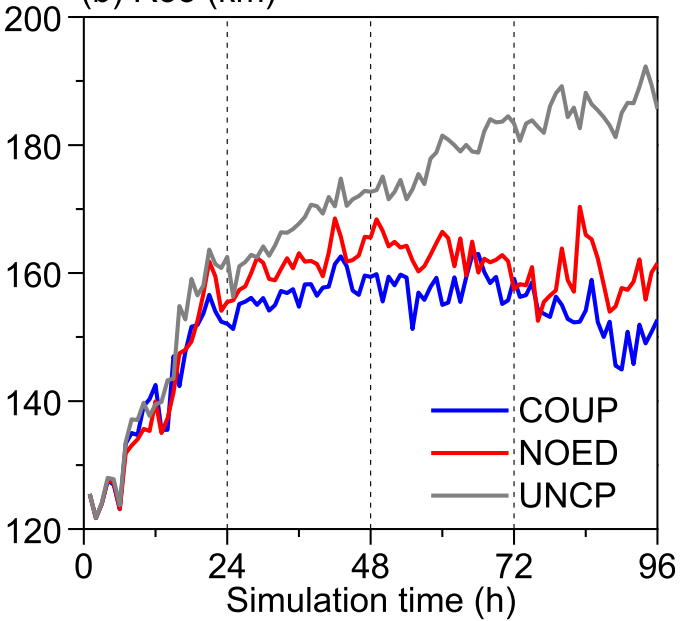

FIG. 1. Time evolution of (a) radius of azimuthally averaged maximum surface wind (RMW; km) and (b) radius of azimuthally averaged hurricane-force $\left(33 \mathrm{~m} \mathrm{~s}^{-1}\right)$ wind $(\mathrm{km})$. Vertical dashed lines denote simulation times of 24,48 , and $72 \mathrm{~h}$.

radial pressure gradient is slightly reduced by the cold wake (Fig. 2b), corresponding to a marginally smaller RMW in UNCP than in NOED; with the increasing radial pressure gradient difference, the RMW discrepancy between UNCP and NOED is enlarged steadily afterward. The enlargement of RMW by the cold-core eddy and cold wake implies diminished inward transport of angular momentum, consistent with the reduction of surface wind speed (Fig. 2).

UNCP shows the largest storm size as expected, with the R33 being $20 \%$ larger than that in NOED. The R33 difference between COUP and NOED occurs earlier (a) COUP-NOED

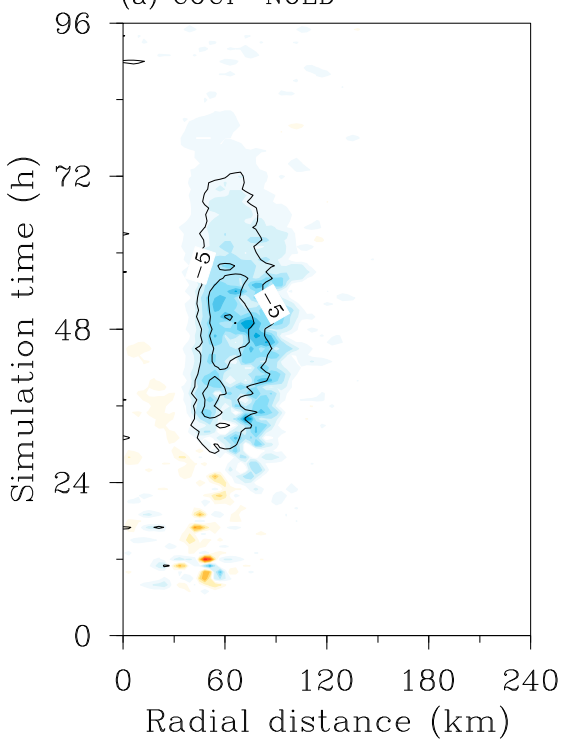

(b) NOED-UNCP

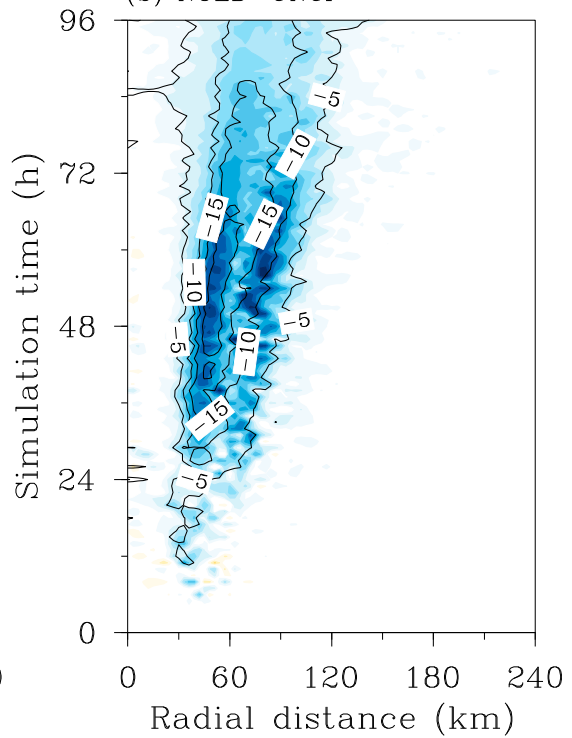

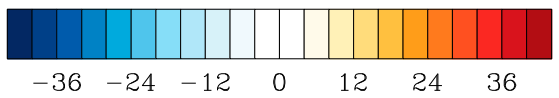

FIG. 2. Hovmöller diagram of azimuthally averaged differences of surface radial pressure gradient $\left(10^{-3} \mathrm{~Pa} \mathrm{~m}^{-1}\right.$; shaded) and surface wind $\left(\mathrm{m} \mathrm{s}^{-1}\right.$; contours) with a contour interval of $5 \mathrm{~m} \mathrm{~s}^{-1}$ for (a) COUP-NOED and (b) NOED-UNCP. The outmost contour of surface wind is $-5 \mathrm{~m} \mathrm{~s}^{-1}$. 
than their intensity difference, which is possibly because the outer-core convections are first inhibited with Francisco approaching the cold-core eddy. Another notable feature is that the storm size remains smaller in COUP than NOED even after departure from the eddy, although the intensity has basically recovered in the later stage. This phenomenon also exists in the idealized simulation of Ma et al. (2013b), reflecting that the TC size is weakly related to the intensity in the mature or decaying phase (Chan and Chan 2015).

\section{b. Thermodynamic boundary layer}

Figure 3 shows radial distributions of azimuthal mean HVTH averaged between 36 and $48 \mathrm{~h}$. The thermodynamic boundary layer height, or rather the mixed-layer depth, is well below $1 \mathrm{~km}$, overall decreasing with radius decreasing in all three experiments, consistent with previous observations and simulations (e.g., Zhang et al. 2011; Lee and Chen 2012). The mixed-layer height is shown to be decreased by both the cold-core eddy and cold wake. Due to their combined effects, HVTH is as shallow as $\sim 50 \mathrm{~m}$ in the eye region in COUP. The impact of cold-core eddy tends to be most significant in the eye region, while the azimuthally averaged HVTH is similarly reduced in both inner-core and outer-core regions by the cold wake.

To compare asymmetric changes in mixed-layer depth caused by the cold-core eddy and cold wake, the time evolution of HVTH difference between COUP and NOED, and between NOED and UNCP, is shown in Figs. 4 and 5, respectively. The storm is azimuthally averaged by dividing it into four quadrants in Cartesian coordinates. Although the lowest level of condensation is changed by sea surface cooling, it has played a minor role in modulating the asymmetric characteristics of HVTH (see the online supplementary material). From Fig. 4, the eddy-induced decrease in HVTH is about $100-200 \mathrm{~m}$ in the eye region for all four quadrants, while in the outer region it is more apparent in the northwest and southeast quadrants than in the other two quadrants. HVTH begins to be decreased in the northwest quadrant approximately when the R33 of Francisco passed over the eddy that possesses a radius of $\sim 2^{\circ}$. Then the decrease in HVTH shifts radially inward, and shows a minimum in the eye region in all four quadrants from $\sim 24$ to $48 \mathrm{~h}$. After the TC center encountered the eddy center ( $32 \mathrm{~h})$, the decrease in HVTH appears in the fourth quadrant and shifts radially outward accompanying the TC departure. The decrease in HVTH also appears in the northeast and southwest quadrants, which is because the eddy possesses a relatively large size. The mixed-layer depth decreases quickly by interacting with the eddy and recovers almost immediately after leaving

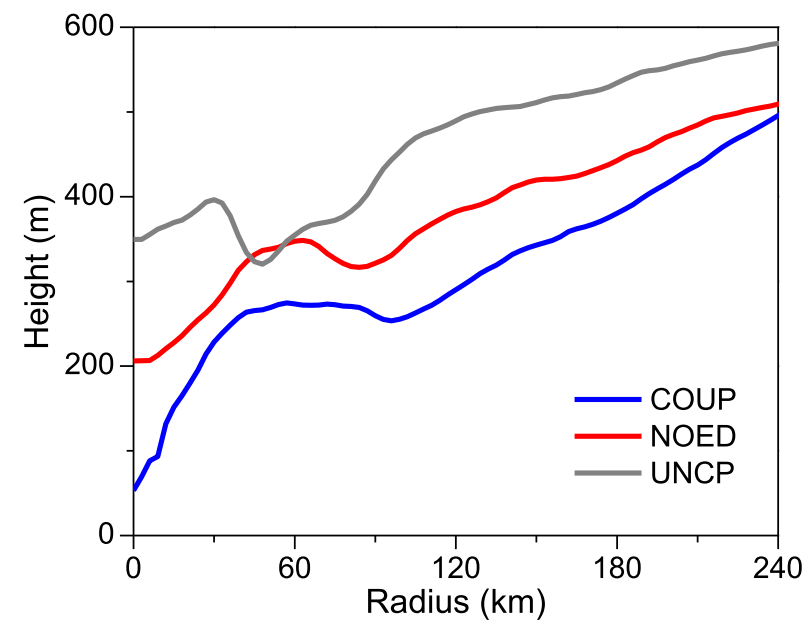

FIG. 3. Radial distribution of azimuthally and temporally averaged depth $(\mathrm{m})$ of the thermodynamic boundary layer between 36 and $48 \mathrm{~h}$.

the eddy, implying that the thermodynamic structures of the boundary layer are quite sensitive to the eddy feedback.

The changes in HVTH caused by the cold wake, indicated from Fig. 5, show different features from those by the cold-core eddy. The HVTH is decreased in the eye region and in the southeast quadrant due to the right-rearward bias of the cold wake. The wake-induced decrease in HVTH also occurs in the northeast and southwest quadrants, due to the vastly spatial distribution of the cold wake (will be illustrated in Fig. 13). However, the cold wake shows almost no influence on the northwest quadrant of the TC, which is a stark difference from the contribution of cold-core eddy. Although the decreases in azimuthally averaged HVTH by cold-core eddy and cold wake are comparable (Fig. 3), the cold wake-induced decrease in HVTH reaches $\sim 300-400 \mathrm{~m}$ in the southeast quadrant, being roughly twice that induced by the cold-core eddy. Besides, the HVTH decrease caused by the cold wake tends to be less significant in the eyewall region. This could not be due to air mixing associated with convective downdrafts (Powell 1990; Barnes and Powell 1995), since the eyewall air is almost saturated as discussed by Zhang et al. (2011). The discrepancies in convection activities between NOED and UNCP may be responsible: the eyewall convection is stronger in the uncoupled run, and the resultant stronger precipitation induces larger diabatic cooling at low levels than the cold wake experiment (not shown). This also implies that the behaviors of thermodynamic boundary layer height reply not only on the ocean state, but also on the intrinsic processes of the TC.

The stable boundary layer is an interesting feature in TCs where convective instabilities are conventionally 
(a) NW quadrant

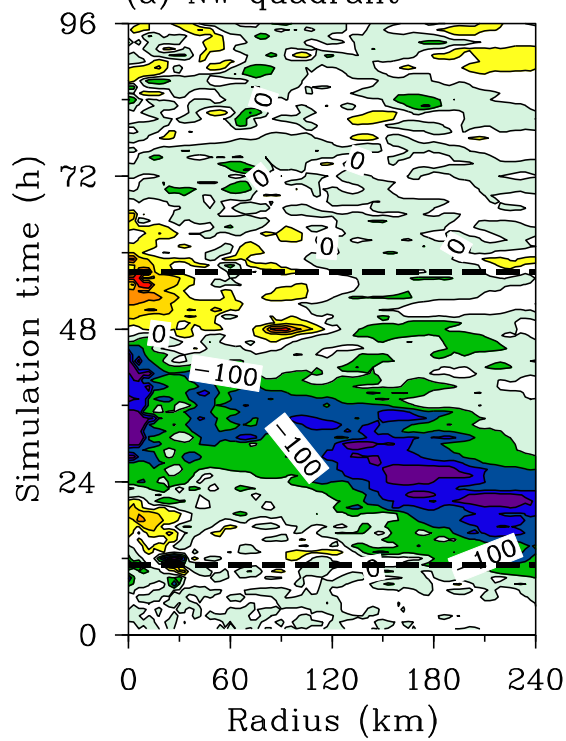

(c) SW quadrant

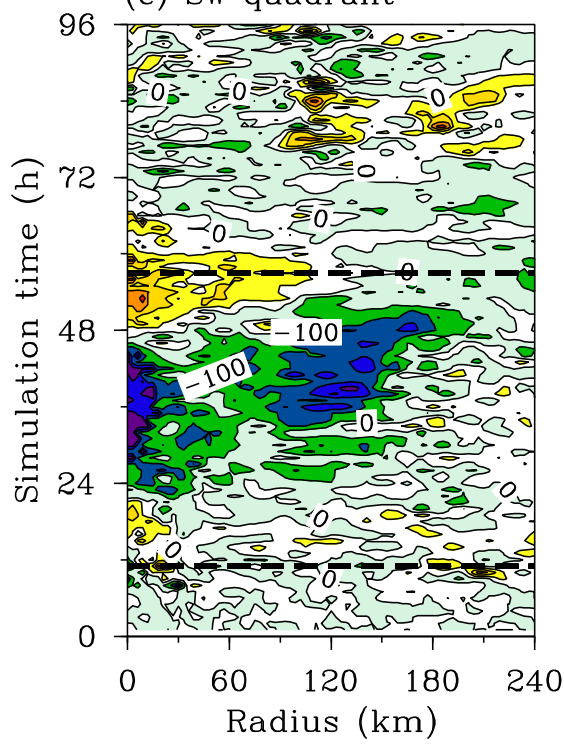

(b) NE quadrant

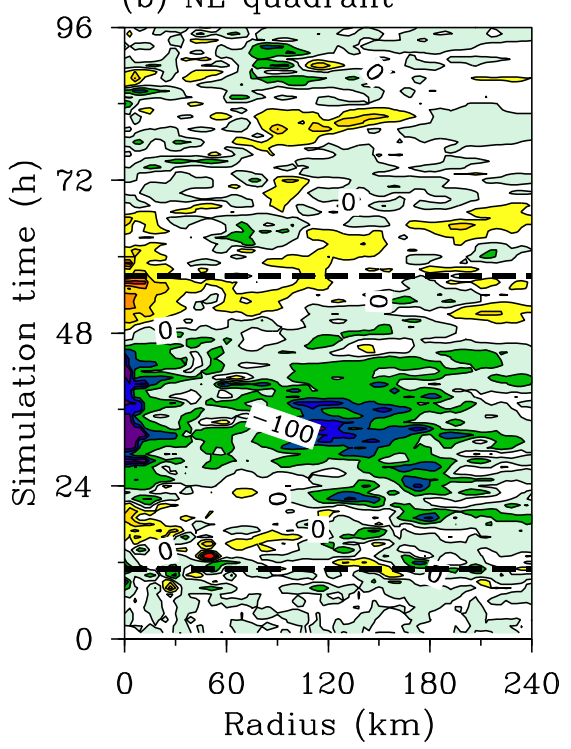

(d) SE quadrant

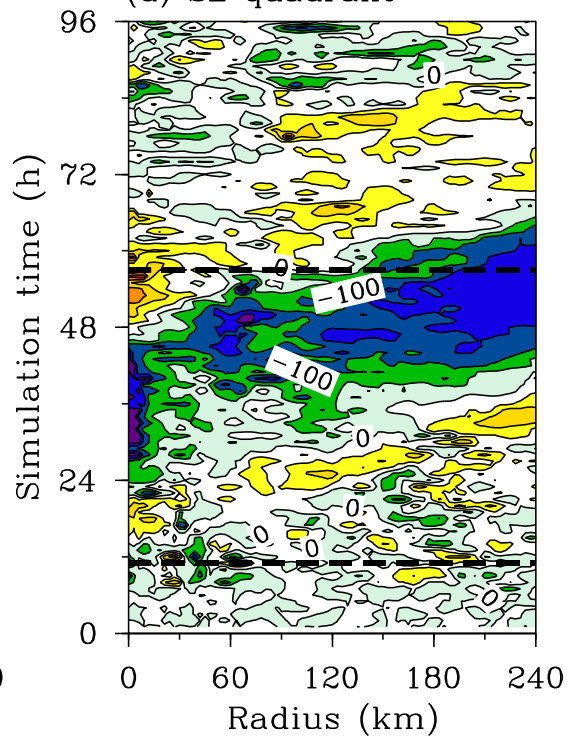

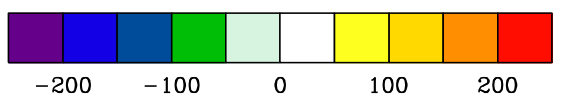

FIG. 4. Hovmöller diagram of azimuthally averaged HVTH difference $(\mathrm{m})$ between COUP and NOED for the (a) northwest, (b) northeast, (c) southwest, and (d) southeast quadrants. The dashed black lines denote the beginning $(11 \mathrm{~h})$ and ending $(57 \mathrm{~h})$ times of interactions between the eddy and R33 of Francisco as in Table 1 of Part I.

considered to dominate. As in Stull (1988), the static stability of the boundary layer is judged according to the value of vertical gradient of $\theta_{v}$. The boundary layer is defined to be unstable as the vertical $\theta_{v}$ gradient is negative at any model level within the boundary layer; otherwise the boundary layer is considered to be stable. This definition basically follows that in Lee and Chen
(2014) for facilitating comparison. Figure 6 shows the distributions of stable boundary layer for COUP, NOED, and UNCP at 24,36 , and $48 \mathrm{~h}$, as well as vertical $\theta_{v}$ gradient between the lowest and second lowest model levels. Negative value of vertical $\theta_{v}$ gradient basically overlaps with the stable boundary layer region, suggesting that the stable boundary layer is formed as a result of direct 
(a) NW quadrant

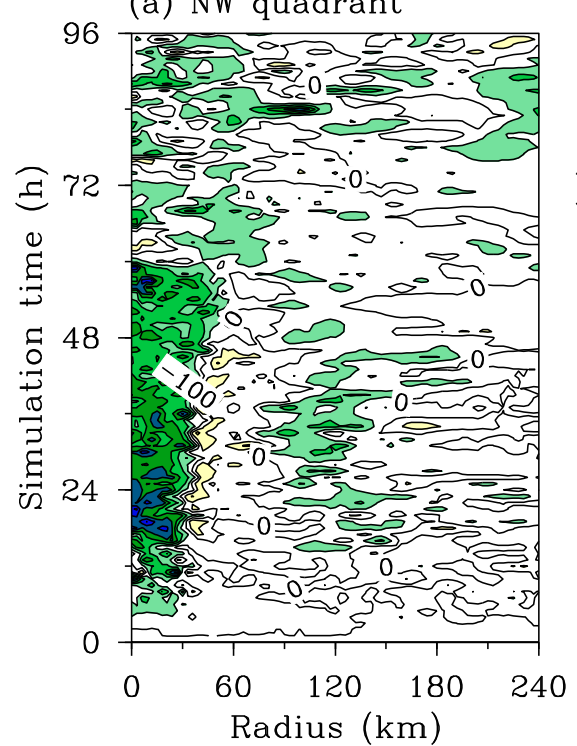

(c) SW quadrant

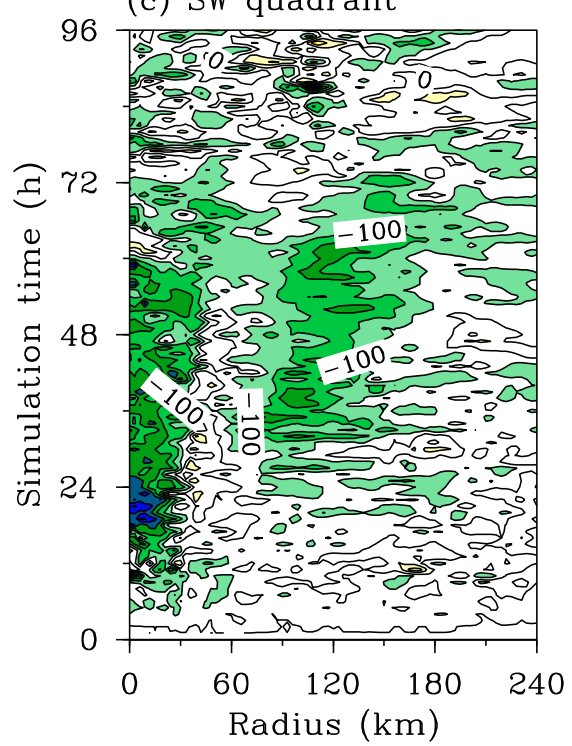

(b) NE quadrant

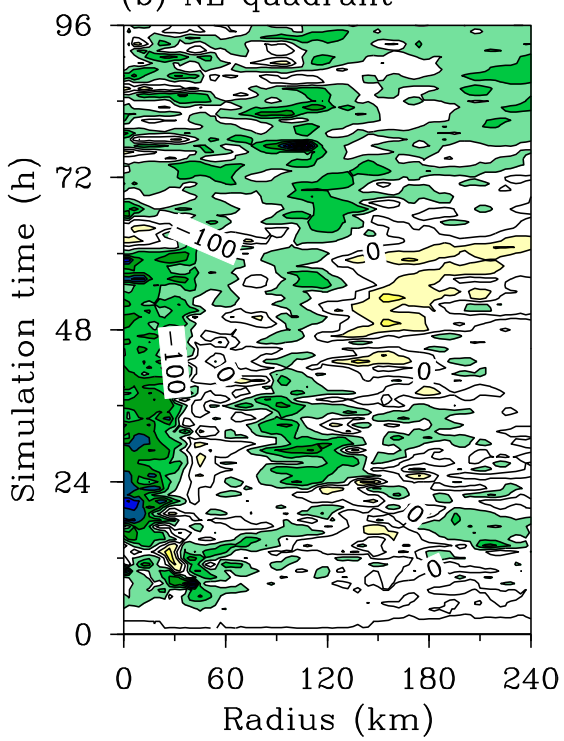

(d) SE quadrant

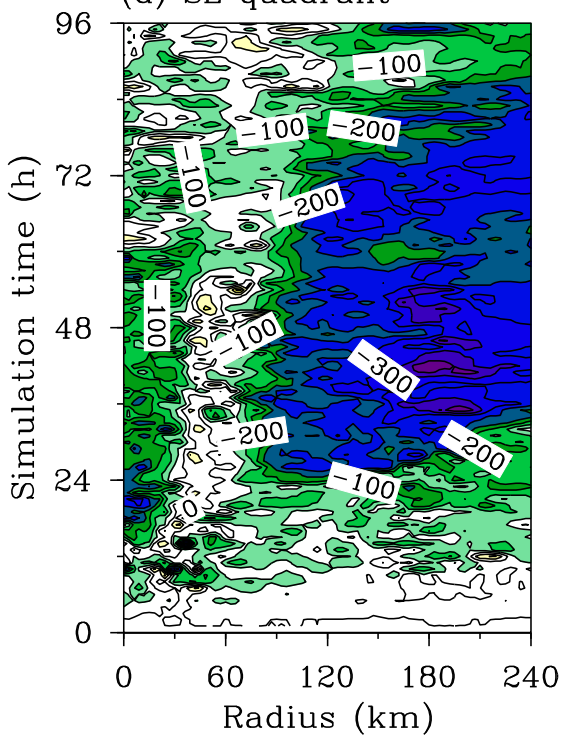

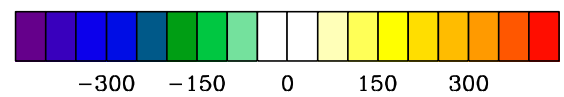

FIG. 5. As in Fig. 4, but for the difference between NOED and UNCP.

cooling from the sea surface. The boundary layer stability in COUP reflects relative locations between Francisco and the eddy: approaching, crossing, and leaving the eddy region (Figs. 6a-c). The stable boundary layer in two coupled experiments exhibits a typical cold wake pattern in the right-rear quadrant after a model integration of $36 \mathrm{~h}$ (Figs. 6b,e), while such as pattern is not evident at $24 \mathrm{~h}$ (Figs. 6a,d), implying that an adjustment time is required for the TC to fully "feel" the oceanic response. This may be why the intensity discrepancy between coupled and uncoupled runs is usually not discernible at the early stage of the simulation (e.g., Zhu et al. 2004; Chen et al. 2010; Ma et al. 2013b; Lee and Chen 2014; Wu et al. 2016; Part I). The boundary layer in the uncoupled run is mostly unstable, except in the north of the storm after $36 \mathrm{~h}$ due to low background SST with the storm translating poleward (Figs. 6g-i). Rather than appearing in a small region as in Lee and Chen (2014), the stable boundary layer covers most of the inner core in COUP due to the combined effect of the cold wake 

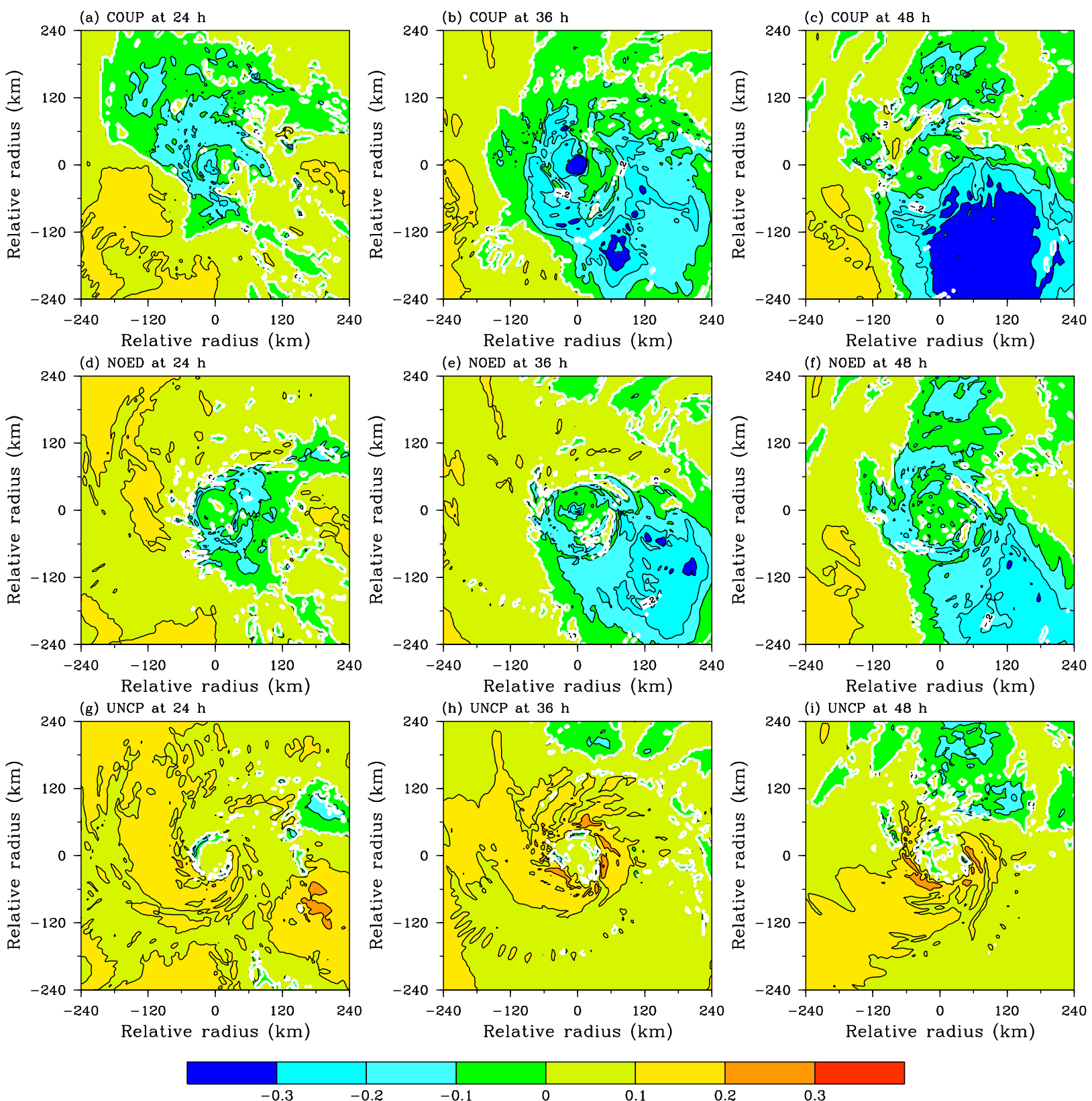

FIG. 6. Plan views of stable boundary layer (white contour) and vertical difference of virtual potential temperature between the lowest and second-lowest model level (shaded) for (a)-(c) COUP, (d)-(f) NOED, and (g)-(i) UNCP at (a),(d),(g) 24, (b),(e),(h) 36, and (c),(f),(i) $48 \mathrm{~h}$.

and eddy, and exists in the whole right-rear quadrant as well as the eye region in NOED at $36 \mathrm{~h}$. This may be because the background SST is lower and the SST decrease is stronger compared to that in Lee and Chen (2014), leading to a larger region where the sea surface is cooler than the surface air.

Black and Holland (1995) found in TC Kerry (1979) that sensible heat fluxes transferred downward into the ocean. The sensible heat fluxes at different atmospheric model levels are diagnosed in this study, by retrieving them based on the potential temperature tendency in the Yonsei University (YSU) boundary layer scheme (Hong et al. 2006). From Fig. 7, downward sensible heat fluxes dominate in COUP at $36 \mathrm{~h}$, when the TC approximately passes over the eddy center; sensible heat fluxes at the lowest model level are also downward in NOED above the cold wake region. In UNCP, however, there are mostly upward sensible heat fluxes. The negative region in COUP 

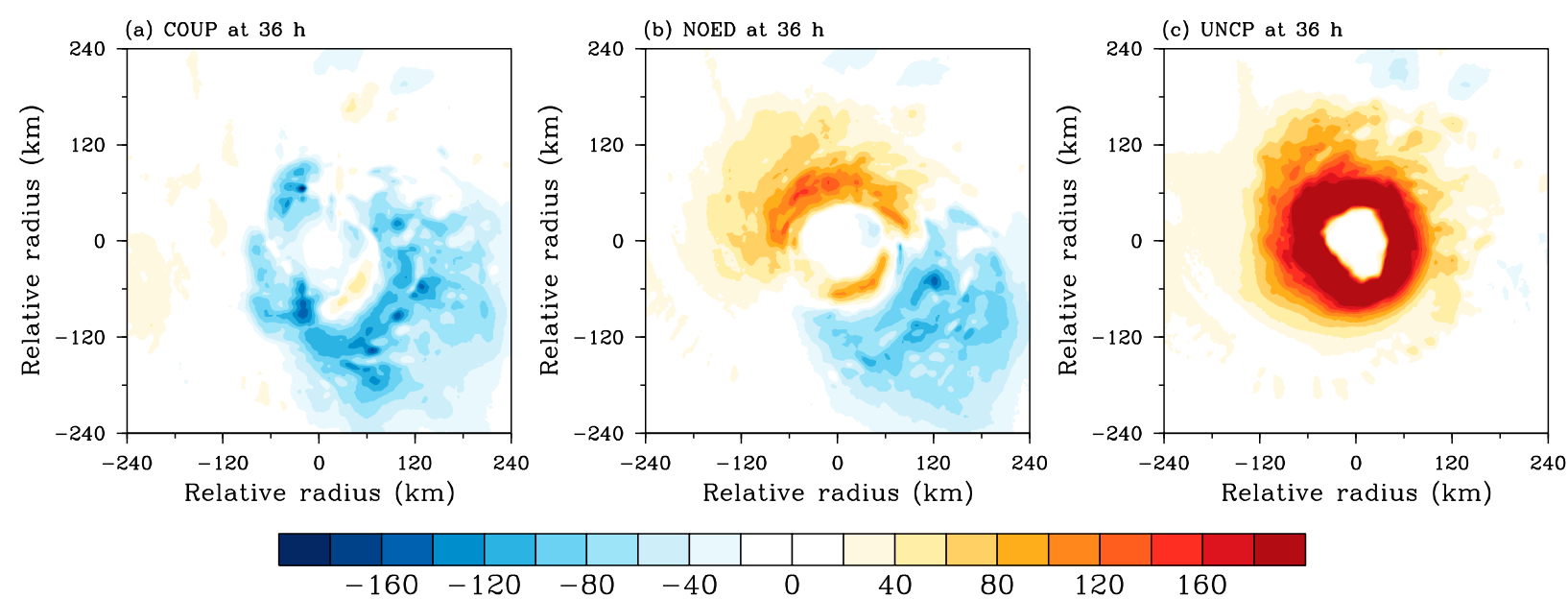

FIG. 7. Plan views of sensible heat fluxes $\left(\mathrm{W} \mathrm{m}^{-2}\right)$ at the lowest atmospheric model level for (a) COUP, (b) NOED, and (c) UNCP at $36 \mathrm{~h}$

and NOED basically overlaps with the feature of cold-core eddy and cold wake, suggesting that downward sensible heat fluxes are an intrinsic feature of the stable boundary layer. Nonetheless, either the upward transfer of sensible heat fluxes in unstable boundary layer or the downward transfer of sensible heat fluxes in stable boundary layer can largely offset the adiabatic cooling of air parcels flowing toward the TC center (Ma et al. 2015). Although the sensible heat fluxes at the lowest model level in NOED are upward in the regions other than above the cold wake, they turns to be downward at the second lowest model level, which is approximately at a height of $100 \mathrm{~m}$ (not shown); the upward transfer of sensible heat fluxes in UNCP vanishes at the third lowest model level ( $200 \mathrm{~m}$; not shown). This difference implies that the oceanic response induces a decrease in the unstable energy not only above the sea surface cooling region, but also in the other regions due to air mixing (Ma et al. 2013a).

The sea-to-air supply of surface enthalpy fluxes is an important source of the equivalent potential temperature $\left(\theta_{e}\right)$ and the convective available potential energy (CAPE), which facilitate the activities of deep convections (e.g., Montgomery et al. 2006; Fang and Zhang 2011). Previous studies suggest that near-surface highenergy air in the eye region could be a prerequisite for the onset of rapid intensification by initiating convection near the inner edge of the eyewall (e.g., Barnes and Fuentes 2010; Rogers 2010; Miyamoto and Takemi 2013; Wang and Heng 2016). To evaluate their roles in the rapid weakening of Francisco, Fig. 8 compares the time-radius evolution of surface equivalent potential temperature $\left(\theta_{e}\right)$ and surface-based CAPE for COUP, its difference from NOED, and the difference between NOED and UNCP. A peak value of surface $\theta_{e}$ occurs in the eye before $24 \mathrm{~h}$ in COUP, when the storm intensifies slightly during this time period; then the surface $\theta_{e}$ in the eye decreases rapidly with crossing over the eddy region. After departure from the eddy, surface $\theta_{e}$ recovers slightly in the eye while decreases in the outer periphery (e.g., after $72 \mathrm{~h}$ ). This signifies an increase of $\theta_{e}$ deficit though the storm continuing to weaken (Fig. 9 in Part I), implying a limitation of the classical positive relationship between the $\theta_{e}$ deficit and central pressure drop (e.g., Malkus and Riehl 1960; Emanuel 1986) in weakening TCs. The surface $\theta_{e}$ is smaller in COUP than that in NOED after about $24 \mathrm{~h}$, primarily in the eye region and near the RMW. The cold wake shows similar but more pronounced and long-lasting effect compared with the cold-core eddy (Fig. 8c). Bhalachandran et al. (2019) proposed that the downward intrusion of low- $\theta_{e}$ air into the boundary layer by strong vertical wind shear leads to the rapid weakening of TC Lehar (2013). Here we show that the intrusion of low- $\theta_{e}$ air into the boundary layer by the cold-core eddy and cold wake contributes to the rapid weakening of Francisco, but oppositely from the surface into the boundary layer.

The surface-based CAPE decreases radially inward as in previous observations (e.g., Molinari et al. 2012). The changes in surface-based CAPE caused by the cold-core eddy and cold wake are mostly pronounced outside the eyewall. In the eye region, however, the decrease in surface-based CAPE caused by the cold-core eddy or cold wake is much delayed relative to the response of storm intensity, indicating that the CAPE in the eye region contribute little to the rapid weakening. This is different from the surface $\theta_{e}$ that closely relates to the intensity change of TCs, and is also in contrast to the positive effect of high surface CAPE in the 
(a) COUP

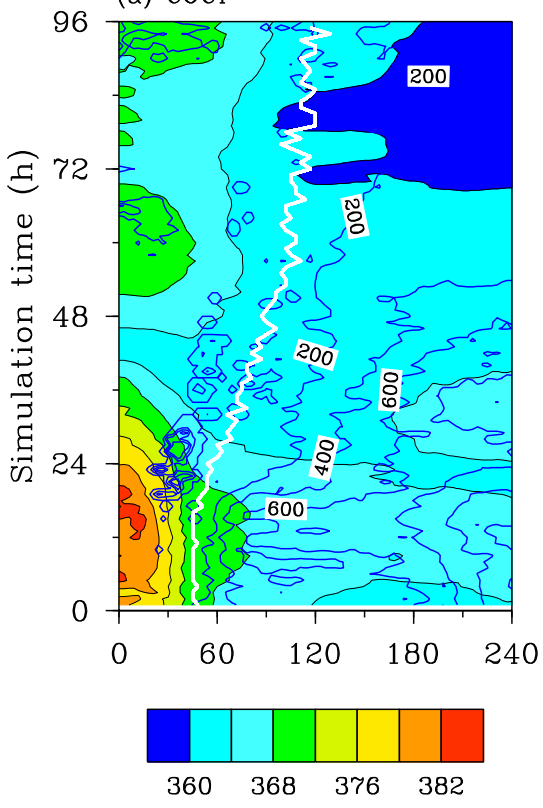

Radial distance ( $\mathrm{km})$ (b) COUP-NOED

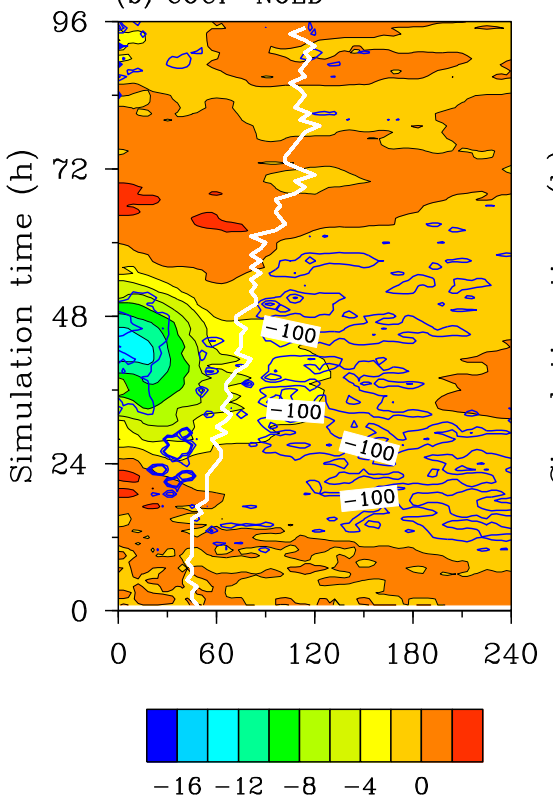

Radial distance ( $\mathrm{km}$ ) (c) NOED-UNCP

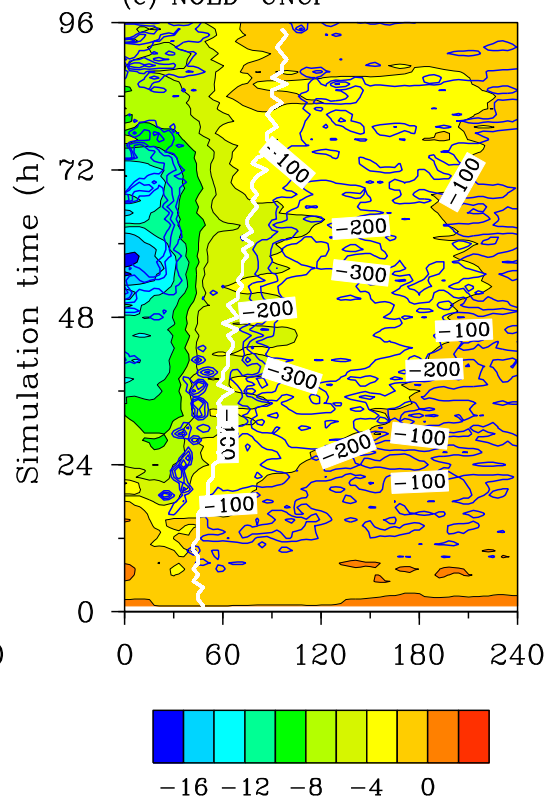

Radial distance (km)

FIG. 8. Hovmöller diagram of azimuthally averaged surface equivalent potential temperature (shaded; K) and surface-based CAPE (blue contours; $\mathrm{J} \mathrm{kg}^{-1}$ ) for (a) COUP, (b) COUP - NOED, and (c) NOED - UNCP. The CAPE is contoured at an interval of $200 \mathrm{~J} \mathrm{~kg}^{-1}$ in (a) and $100 \mathrm{~J} \mathrm{~kg}^{-1}$ in (b) and (c) with only negative values being shown. The solid white curve shows the radius of azimuthally averaged maximum surface wind for (a) COUP, (b) NOED, and (c) UNCP.

eye on initiating the onset of rapid intensification (e.g., Miyamoto and Takemi 2013; Wang and Heng 2016).

Figure 9 compares the diabatic heating rate and inertial stability averaged between 36 and $48 \mathrm{~h}$ for three experiments by dividing the storm into four quadrants. The peak diabatic heating rate is shown to be located inside the radius of maximum wind speed for all experiments. Nonetheless, large discrepancies exist for three experiments in different quadrants. A comparison between NOED and UNCP shows that the diabatic heating is inhibited by the cold wake in all four quadrants, as a result of air mixing between the cooled air parcels and nearby swirling air parcels (Ma et al. 2013a). There are quantitative differences among four quadrants: in UNCP both the northwest and southwest quadrants give the strongest diabatic heating, while in NOED the diabatic heating is the strongest in the northwest quadrant; the northwest quadrant is also the least influenced by the cold wake, possessing the same peak values between NOED and UNCP. This is similar with the behavior of thermodynamic boundary layer depth (Fig. 5). As for the cold-core eddy, the diabatic heating is also reduced in all four quadrants by comparing COUP with NOED. A notable feature is that the diabatic heating in the northwest quadrant is considerably inhibited, with the peak values reduced by roughly a half. The different roles of cold-core eddy and cold wake in modulating diabatic heating in the northwest quadrant correspond well with the differences of surface enthalpy fluxes among three experiments (not shown), reflecting that although the right-rearward biased cold wake may be the least influential for the left-front side of the TC, the cold-core eddy can affect that region directly during the TC-eddy interaction. The inertial stability is shown to be the largest in UNCP, followed by NOED, while it is the smallest in COUP for all four quadrants. This suggests that the cold-core eddy and cold wake not only diminish the diabatic heating, but also decrease its dynamical efficiency in spinning up tangential wind in the inner core (Vigh and Schubert 2009; Rogers et al. 2013).

\section{c. Dynamic boundary layer}

The dynamic definition regarding the frictional inflow layer as the boundary layer has difficulties in deriving the accurate depth. Here we take HRAD as the height where radial velocity is $10 \%$ of the peak inflow following Zhang et al. (2011). Figure 10 compares the radial distributions of HRAD in four quadrants for COUP, NOED, and UNCP. Approximately inside the RMW, HRAD increases radially outward, corresponding well with previous theoretical and observational studies 


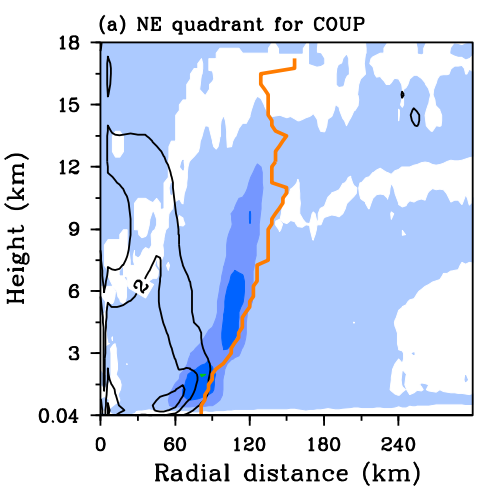

(d) NW quadrant for COUP

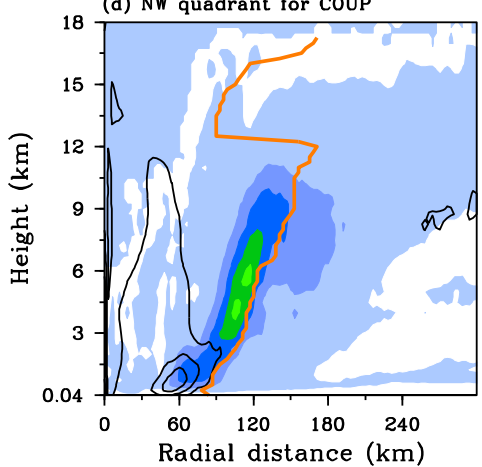

(g) SW quadrant for couP
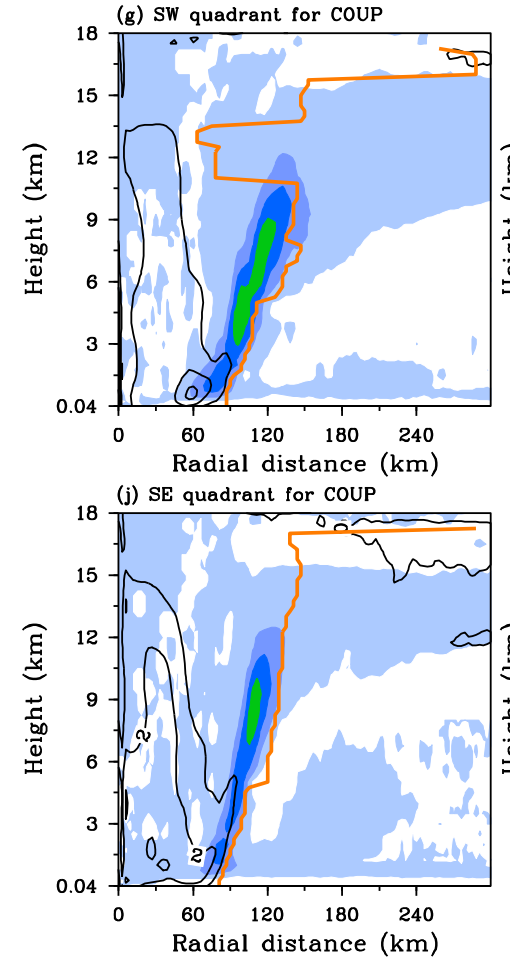

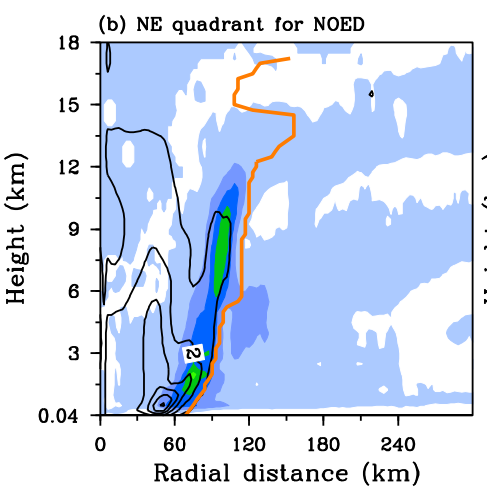

(e) NW quadrant for NOED

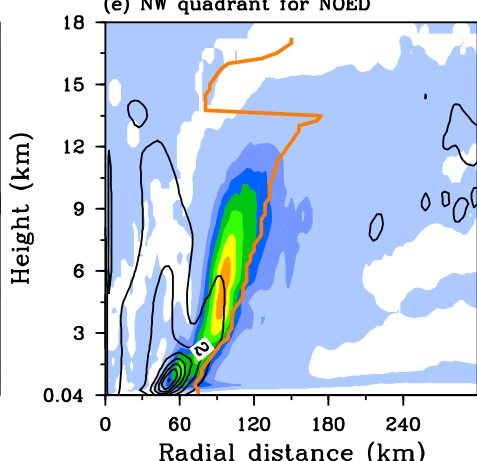

(h) SW quadrant for NOED

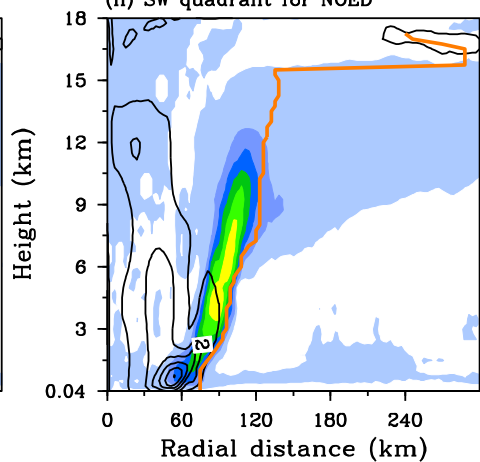

(k) SE quadrant for NOED

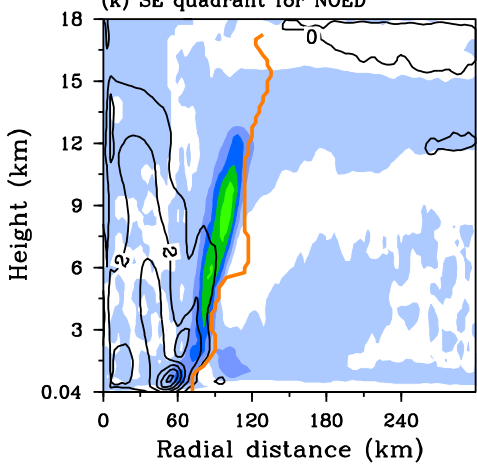

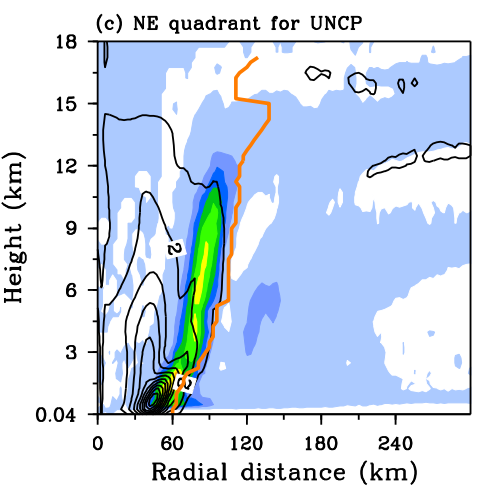

f) NW quadrant for UNCP
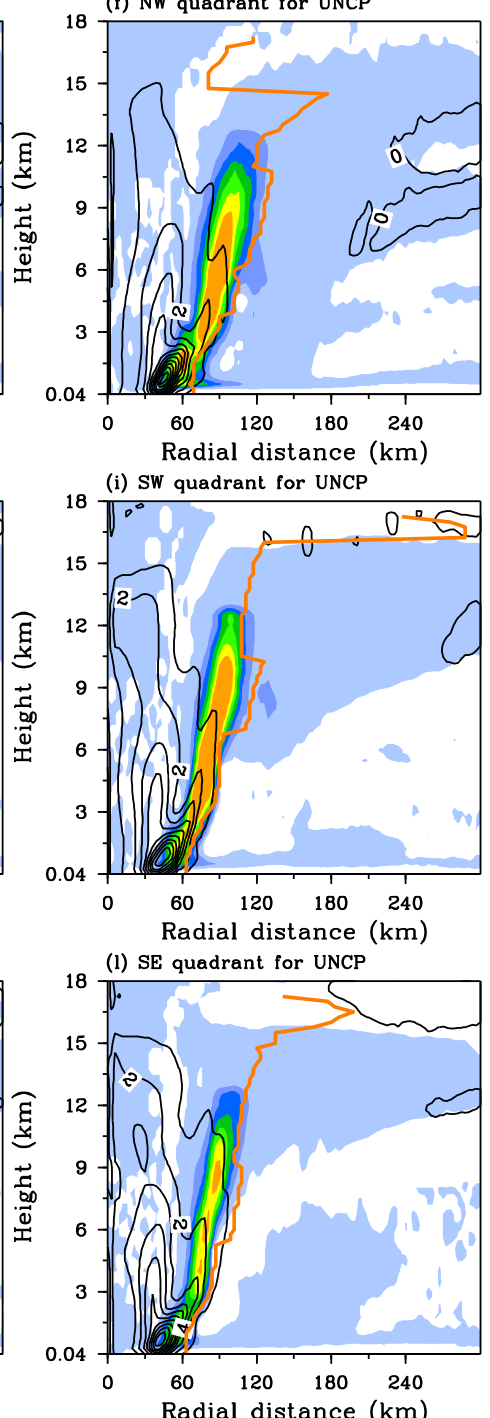

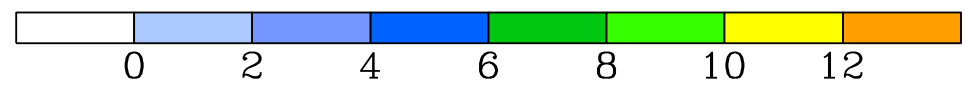

FIG. 9. Height-radius cross sections of azimuthally and temporally averaged microphysical diabatic heating rate (shaded; $10^{-3} \mathrm{~K} \mathrm{~s}^{-1}$ ) and inertial stability (contours; $10^{-6} \mathrm{~s}^{-2}$; interval: $1 \times 10^{-6} \mathrm{~s}^{-2}$ ) between 36 and $48 \mathrm{~h}$ for (left) COUP, (center) NOED, and (right) UNCP and for the (a)-(c) northeast, (d)-(f) northwest, (g)-(i) southwest, and (j)-(l) southeast quadrants. 

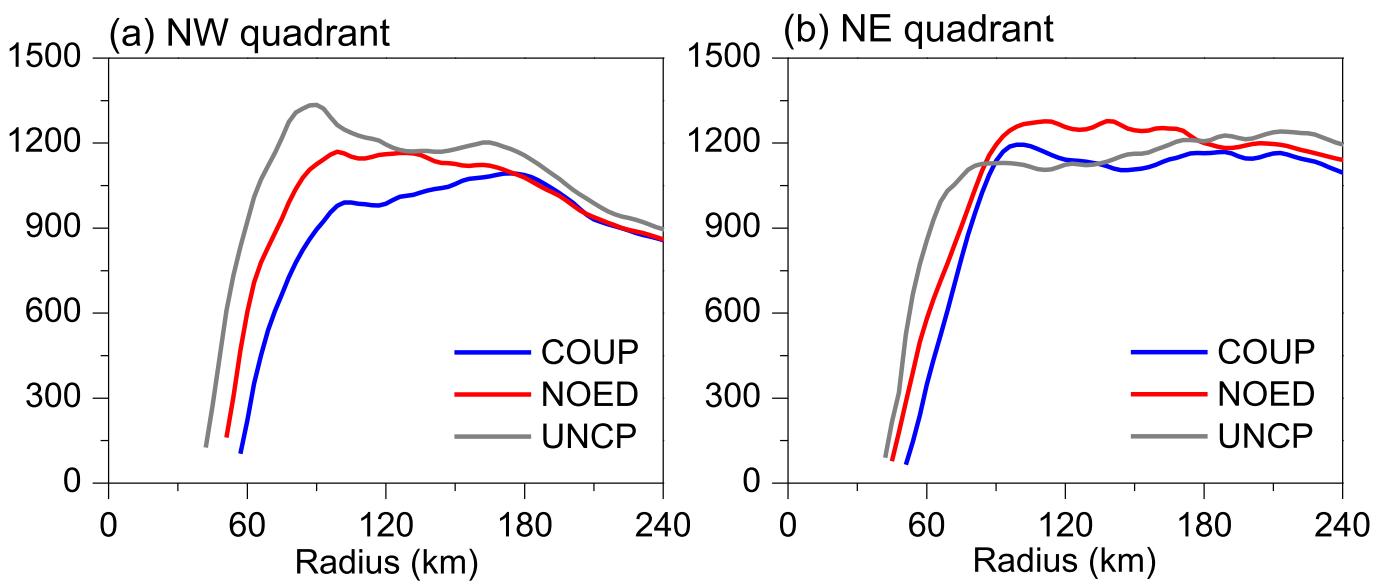

(c) SW quadrant

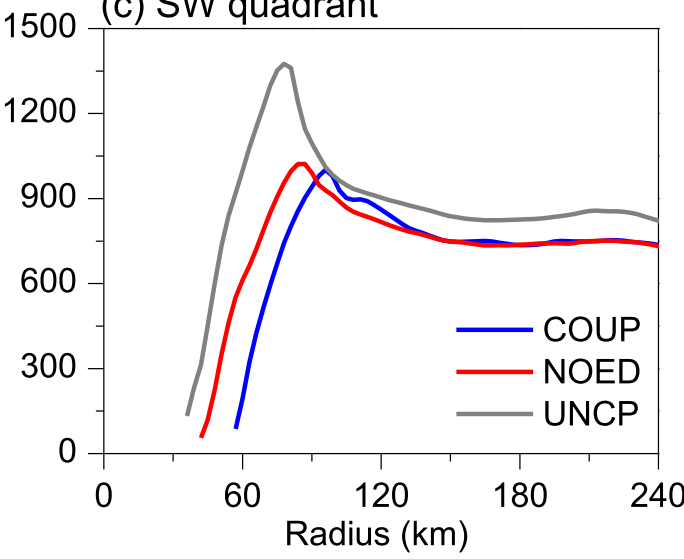

(d) SE quadrant

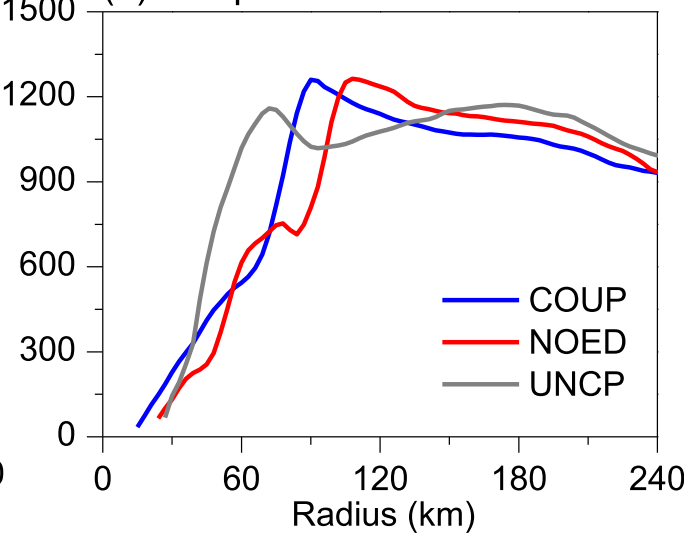

FIG. 10. Radial distribution of azimuthally and temporally averaged depth $(\mathrm{m})$ of the dynamic boundary layer between 36 and $48 \mathrm{~h}$ for the (a) northwest, (b) northeast, (c) southwest, and (d) southeast quadrants.

(Kepert 2001; Zhang et al. 2011). However, with increasing the radius further, HRAD keeps mostly invariant or even decreases in some quadrants (Figs. 10a,c,d). This may be linked with the feature that the radial inflow attains a maximal strength near the RMW. Although the HRAD is the deepest in UNCP and shallowest in COUP inside the RMW for the northwest, northeast, and southwest quadrants, it shows an inconsistent relationship for the southeast quadrant, with COUP producing comparable or even slightly larger HRAD than NOED. This reveals that the dynamic boundary layer height does not fully comply with the influence of oceanic feedback.

Figure 11 compares surface tangential and radial winds in four quadrants temporally averaged between 36 and $48 \mathrm{~h}$. The peak values of both tangential and radial winds in all four quadrants are consistent with the discrepancies in storm intensities among three experiments. The tangential wind is quite symmetrically distributed, while the radial wind exhibits large asymmetry in different quadrants. This could be because the acceleration of radial wind is closely related to radial pressure gradient force that is modulated by diabatic heating, while the acceleration of tangential wind depends indirectly on the radial wind via the generalized Coriolis term (Gopalakrishnan et al. 2011, 2013). From Fig. 11b, the radial wind is the strongest in the northwest quadrant for all three experiments, corresponding well with the strongest diabatic heating rate in the northwest quadrant (Fig. 9). In UNCP, the radial winds in the other three quadrants show comparable though slightly smaller values than that of the northwest quadrant. In NOED, however, the radial wind is notably reduced in the southeast and southwest quadrants, especially the southeast quadrant where the cold wake prevails. Relative to NOED, the difference of surface radial wind between the northeast and northwest quadrants is narrowed in COUP, which is probably related to diminished diabatic heating rate in the northwest quadrant due to the coldcore eddy.

Lee and Chen (2014) found in their simulation that the inflow angle and therefore the inflow strength tend to be larger over the cold wake, which they attributed to be a consequence of stable boundary layer associated with weak turbulent mixing. However, this explanation 
(a) Tangential wind $\left(\mathrm{m} \mathrm{s}^{-1}\right)$

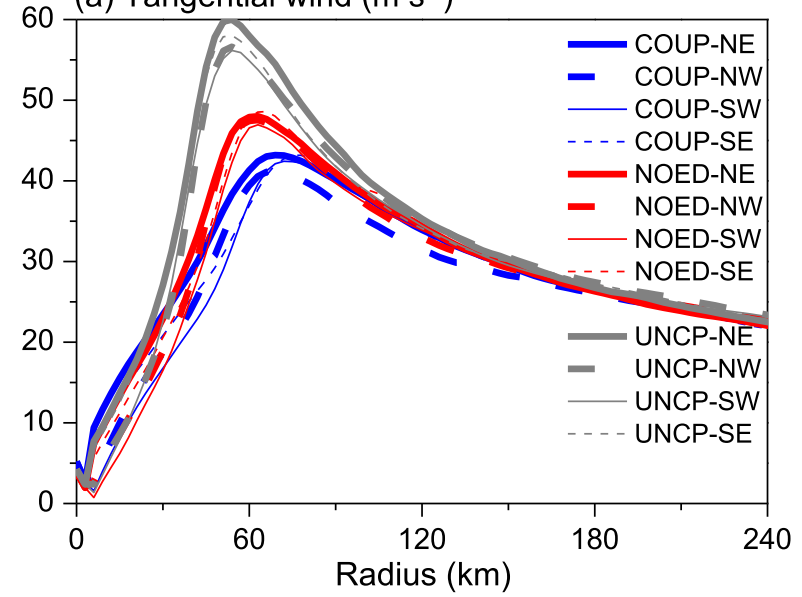

(b) Radial wind $\left(\mathrm{m} \mathrm{s}^{-1}\right)$

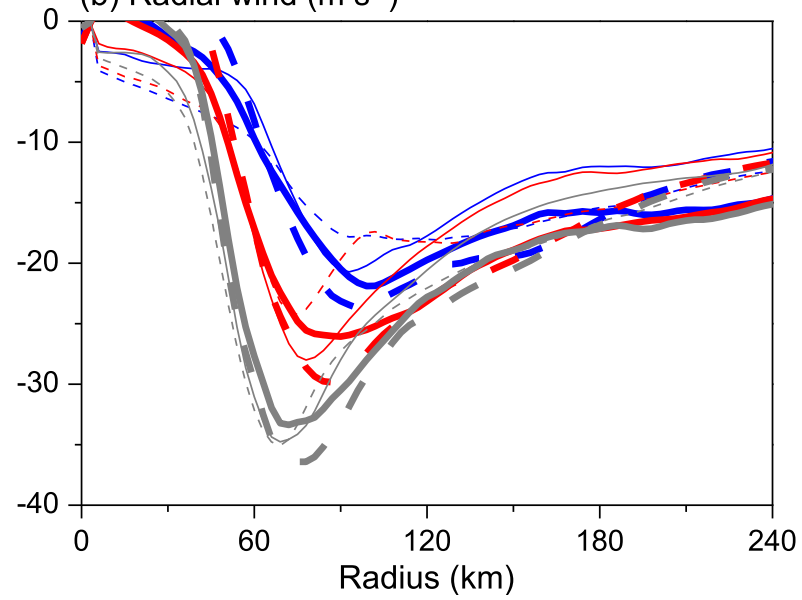

FIG. 11. Radial distribution of azimuthally and temporally averaged (a) surface tangential wind $\left(\mathrm{m} \mathrm{s}^{-1}\right)$ and (b) surface radial wind $\left(\mathrm{m} \mathrm{s}^{-1}\right)$ between 36 and $48 \mathrm{~h}$ for all four quadrants.

seems to be not plausible in this case. The cold wake inhibits the convection activities and therefore diabatic heating above it (Fig. 9), being unfavorable for the acceleration of radial inflow (Shapiro and Willoughby 1982; Pendergrass and Willoughby 2009). This process can be confirmed from the weakened radial winds in the southeast quadrant in NOED relative to UNCP (Fig. 11b). Considering that the tangential wind is relatively symmetrically distributed, the cold wake would lead to a decrease in the ratio of radial wind to tangential wind, implying a decrease of inflow angle.

The plan views of surface radial wind in COUP as well as the radial wind changes caused by cold-core eddy and cold wake are exhibited in Fig. 12. The COUP shows a progressively broadened eye size and weakening of radial wind by interacting with the cold-core eddy. Before the eye approaching the eddy, the radial wind is moderately reduced in northwest of the storm (Fig. 12d).
This discrepancy is enlarged as the storm center crosses over the eddy center, while in the right rear of the storm the radial wind in COUP remains slightly stronger than that in NOED (Fig. 12e). After that, the storm center has left from the eddy, weaker radial wind occurs in the right-rear quadrant (Fig. 12f). At $60 \mathrm{~h}$ the radial wind in COUP is mostly smaller than that in NOED (not shown). The cold wake-induced weakening of radial wind is first evident in the right-rear quadrant (Fig. 12g); this region expands cyclonically and covers most of the TC inner core (Figs. 12h,i). These results suggest that the asymmetry of radial wind relates closely to the presence of cold wake and cold-core eddy. However, the changes in tangential wind caused by cold wake or cold-core eddy seem to be randomly distributed (not shown), indicating that the asymmetric characteristics of tangential wind are not sensitive to the SST response.

The sensitivity of radial wind asymmetry but insensitivity of tangential wind asymmetry to sea surface cooling implies a change in surface inflow angle. Corresponding to Fig. 12, the plan views of surface wind vectors for COUP, NOED, and UNCP at 24, 36, and $48 \mathrm{~h}$ are shown in Fig. 13, superimposed by SST difference caused by the cold-core eddy and cold wake, respectively. The time evolution of sea surface cooling region in Figs. $13 \mathrm{a}-\mathrm{c}$ reflects the relative location between the storm and the eddy, while the cold wake is located at the right rear of the storm (Figs. 13d-f). In COUP there is an evident outward turning of surface wind at 36 and $48 \mathrm{~h}$ compared to that in NOED. The smaller inflow angle caused by cold-core eddy exists in the west and southwest of the TC, where there is evident SST decrease induced by the cold-core eddy. A comparison between NOED and UNCP shows that the cold wake also induces remarkably outward turning of surface wind flow, which is initially remarkable in the right rear of the TC and expands to other quadrants at $48 \mathrm{~h}$ (Fig. 13f). This result is clearly contrary to that in Lee and Chen (2014) and Wu et al. (2016), in which the mean inflow angle is decreased by the cold wake while the inflow angle is increased above and near the cold wake. The different conclusion from those studies could be related to different evolutionary stages of TCs, since this study focuses on the weakening period while their storms are basically in steady states [Fig. 11 in Lee and Chen (2014) and Fig. 13 in Wu et al. (2016)]. Nonetheless, this issue may be worth further studies with more detailed comparisons for various TC cases in different evolution phases.

The changes in surface inflow angles caused by the cold wake and cold-core eddy can be more quantitatively reflected from Table 1 . The area-averaged inflow 


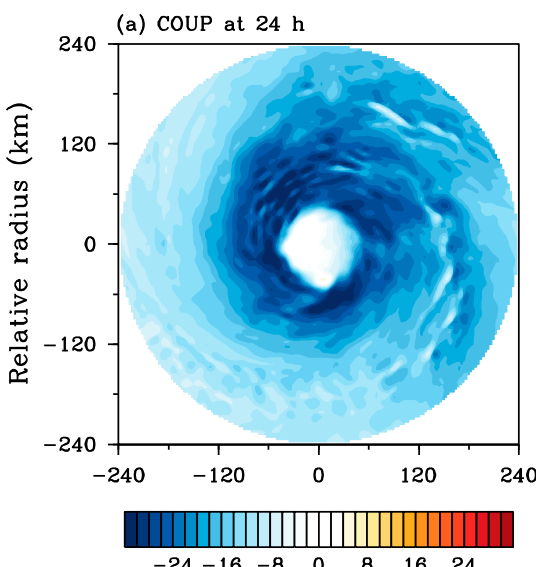

Relative radius $(\mathrm{km})$ (d) COUP-NOED at $24 \mathrm{~h}$

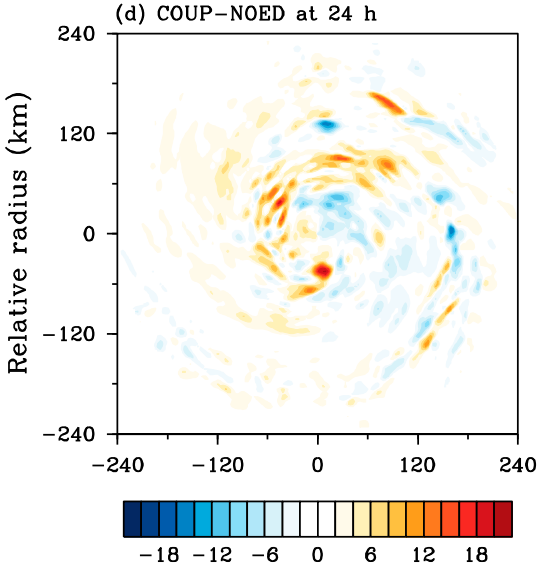

Relative radius $(\mathrm{km})$

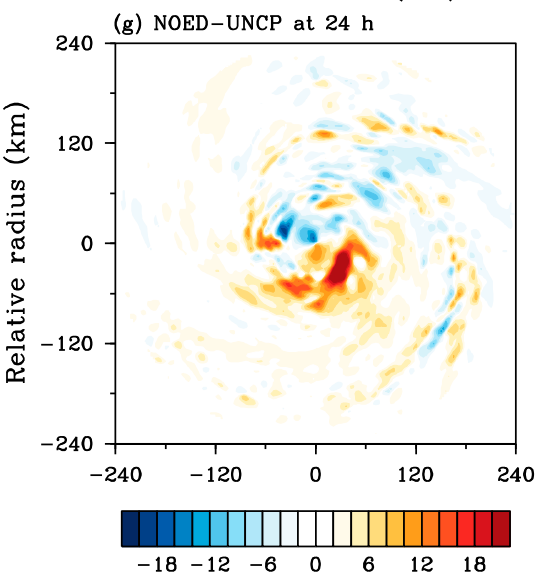

Relative radius $(\mathrm{km})$

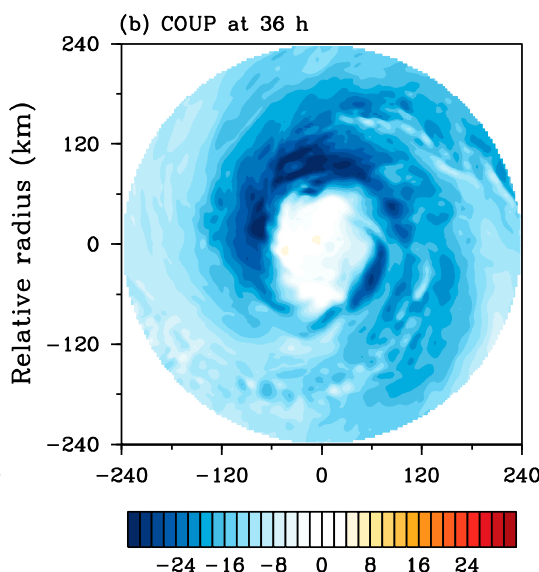

Relative radius $(\mathrm{km})$ (e) COUP-NOED at $36 \mathrm{~h}$

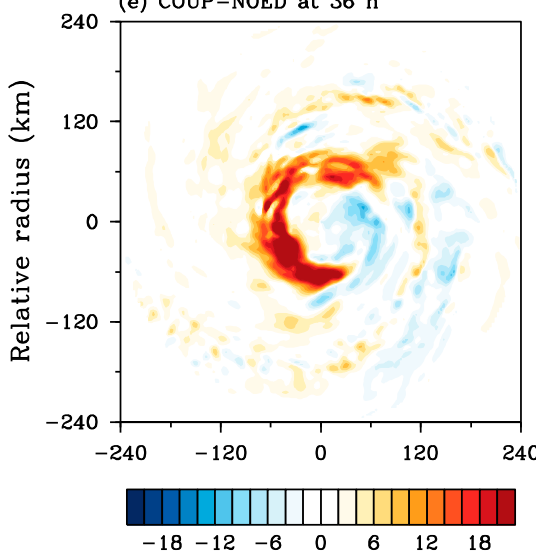

Relative radius ( $\mathrm{km})$ (h) NOED-UNCP at $36 \mathrm{~h}$

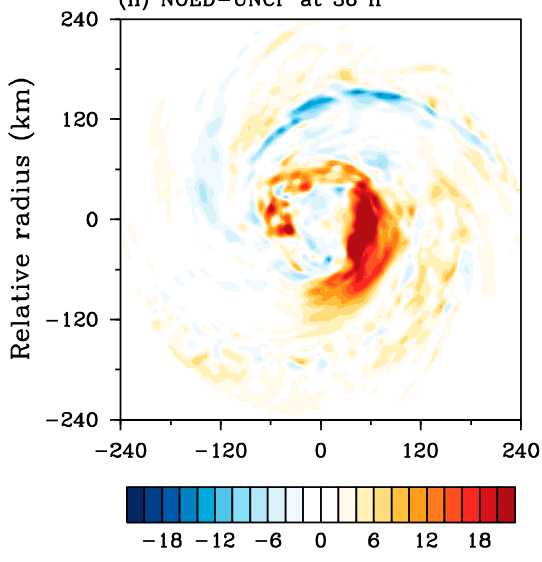

Relative radius $(\mathrm{km})$

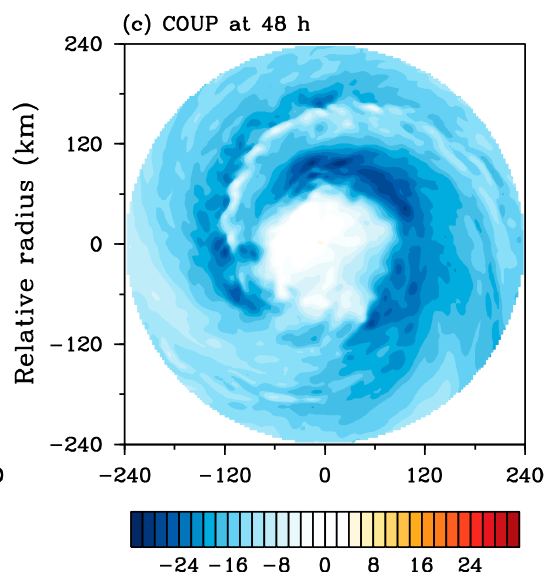

Relative radius $(\mathrm{km})$

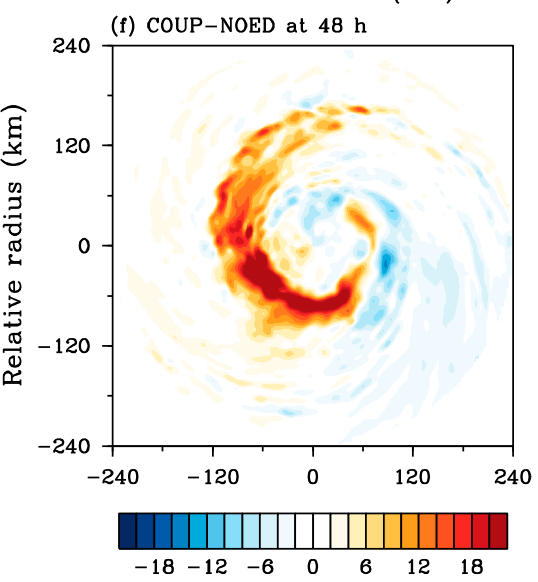

Relative radius $(\mathrm{km})$

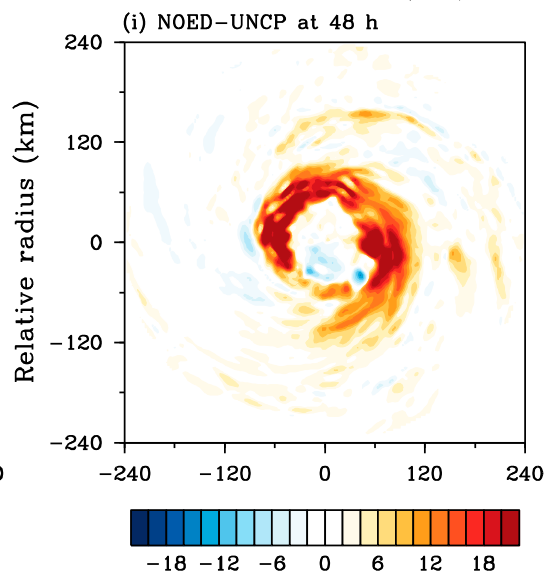

Relative radius $(\mathrm{km})$

FIG. 12. Plan views of surface radial wind $\left(\mathrm{m} \mathrm{s}^{-1}\right.$ ) for COUP at (a) 24 , (b) 36 , and (c) $48 \mathrm{~h}$, and surface radial wind difference (m s${ }^{-1}$ ) between COUP and NOED at (d) 24, (e) 36, and (f) 48 h, and between NOED and UNCP at (g) 24, (h) 36, and (i) 48 h. 

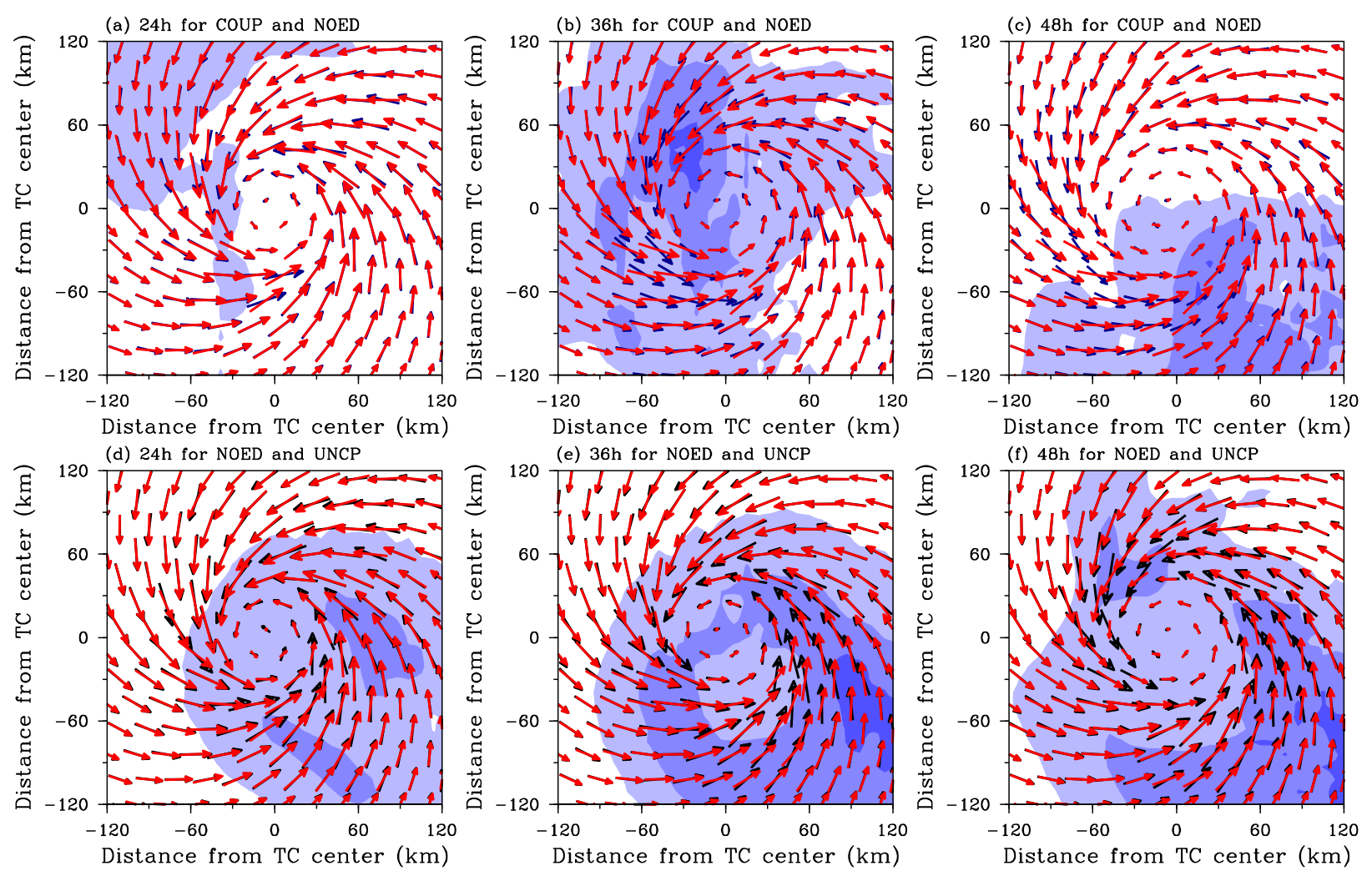

$-3$

$-2$

FIG. 13. Plan views of SST difference (K) and surface wind vectors for (a)-(c) COUP and NOED, with blue vectors representing COUP and red vectors representing NOED, and (d)-(f) NOED and UNCP, with red vectors representing NOED and black vectors representing UNCP at (a),(d) 24, (b),(e) 36, and (c),(f) $48 \mathrm{~h}$.

angle within a radius of $120 \mathrm{~km}$ is the smallest in COUP, followed by NOED, and UNCP gives the largest inflow angle, with their difference ranging from $2.3^{\circ}$ to $4.7^{\circ}$. Of interest is that over the eddy-induced cooling region, the inflow angle is reduced by $4.8^{\circ}(36 \mathrm{~h})$ and $3.9^{\circ}(48 \mathrm{~h})$ by comparing COUP with NOED; over the cold wake region, the inflow angle is reduced by $3.9^{\circ}(36 \mathrm{~h})$ and $5.3^{\circ}$ ( $48 \mathrm{~h}$ ) by comparing NOED with UNCP. The inflow angle difference averaged over the eddy or the cold wake region is uniformly larger than that over the whole domain, indicating that the surface wind inflow angle is reduced especially over the sea surface cooling region.

Based on the case of Typhoon Choi-Wan (2009), Lee and Chen (2014) speculated that the stable boundary layer led to a decrease in turbulent mixing, which induced smaller tangential wind, larger gradient wind imbalance, and eventually stronger inflow motion and larger inflow angle. In essence this explanation stresses the dominance of the decrease in the Coriolis and centrifugal forces on modulating the inflow motion. To understand why the inflow angle and strength are reduced by sea surface cooling in this case, the surface wind tendency equation is diagnosed. The radial and tangential wind equations are written as

$$
\begin{aligned}
& \frac{d u}{d t}=\left(f v+\frac{v^{2}}{r}\right)-\frac{1}{\rho} \frac{\partial p}{\partial r}+F_{r}, \\
& \frac{d v}{d t}=-u\left(f+\frac{v}{r}\right)-\frac{1}{r \rho} \frac{\partial p}{\partial \lambda}+F_{v},
\end{aligned}
$$

TABLE 1. Area-averaged inflow angle. The cold-core eddy (CCE) region and cold wake region refer to the areas of SST difference being smaller than $-1^{\circ} \mathrm{C}$ between COUP and NOED and between NOED and UNCP, respectively, within a radius of $120 \mathrm{~km}$ from the storm center. The pairs of boldface values show the two experiments that should be compared.

\begin{tabular}{cccc}
\hline \hline Expt & 120-km radius & CCE region & Cold wake region \\
\hline COUP $(36 \mathrm{~h})$ & 22.3 & $\mathbf{2 1 . 3}$ & 18.3 \\
NOED $(36 \mathrm{~h})$ & 26.0 & $\mathbf{2 6 . 1}$ & $\mathbf{2 2 . 6}$ \\
UNCP $(36 \mathrm{~h})$ & 28.3 & 27.3 & $\mathbf{2 6 . 5}$ \\
COUP $(48 \mathrm{~h})$ & 20.3 & $\mathbf{1 9 . 6}$ & 18.8 \\
NOED $(48 \mathrm{~h})$ & 23.9 & $\mathbf{2 3 . 5}$ & $\mathbf{2 2 . 1}$ \\
UNCP $(48 \mathrm{~h})$ & 28.6 & 26.9 & $\mathbf{2 7 . 4}$ \\
\hline
\end{tabular}


where

$$
\frac{d}{d t}=\frac{\partial}{\partial t}+u \frac{\partial}{\partial r}+\frac{v}{r} \frac{\partial}{\partial \lambda}+w \frac{\partial}{\partial z}
$$

$v, u$, and $w$ denote tangential, radial, and vertical winds, respectively; $\rho$ is air density and $p$ is pressure; $t, r, z$, and $\lambda$ are time, radius, height, and azimuth, respectively; and $F_{r}$ and $F_{v}$ are the radial and tangential components of frictional force, output directly from the boundary layer scheme. The three right-hand-side terms in Eq. (1) are the sum of the Coriolis and centrifugal forces, the radial pressure gradient force, and the radial frictional stress. The three right-hand-side terms in Eq. (2) are the generalized Coriolis term (Smith et al. 2009), the azimuthal pressure gradient contribution, and the tangential frictional stress.

As inferred from Eq. (1), the acceleration of radial inflow depends not only on the sum of the Coriolis and centrifugal forces that is related to storm intensity, but also on the radial pressure gradient force that could be related to diabatic heating. Figure 14 shows plan views of vertically averaged diabatic heating rate and asymmetric surface pressure perturbation in COUP averaged between 36 and $48 \mathrm{~h}$. The diabatic heating is relatively strong in the northwest quadrant while is less pronounced in the east of the storm. Correspondingly, the asymmetric surface pressure is the lowest in the northwest quadrant with negative perturbation present, and shows the largest value in the southeast quadrant where the diabatic heating is weak. This suggests that an increase in diabatic heating tends to cause a drop in the surface pressure. NOED and UNCP give a consistent relationship between the diabatic heating and asymmetric surface pressure perturbation (not shown). Therefore, both the cold wake and cold-core eddy are capable of modulating the radial pressure gradient force via changing the diabatic heating rate.

A comparison of left-hand-side term and the sum of right-hand-side terms in Eq. (1) shows that the total differential of radial wind can be basically represented by the budget calculations (Fig. 15a). From the Lagrangian perspective, the surface air parcel accelerates inward as the sum of three right-hand-side terms in Eq. (1) is negative, and its radial wind reaches a maximum when the total radial force turns to be positive (outward). A comparison of three experiments shows that the acceleration of radial wind is the slowest and the radius of maximal radial wind is the largest in COUP, followed by NOED, while UNCP produces the strongest convergence and the fastest acceleration. This indicates that both the cold wake and the cold-core eddy act to inhibit the inward acceleration of radial flow.
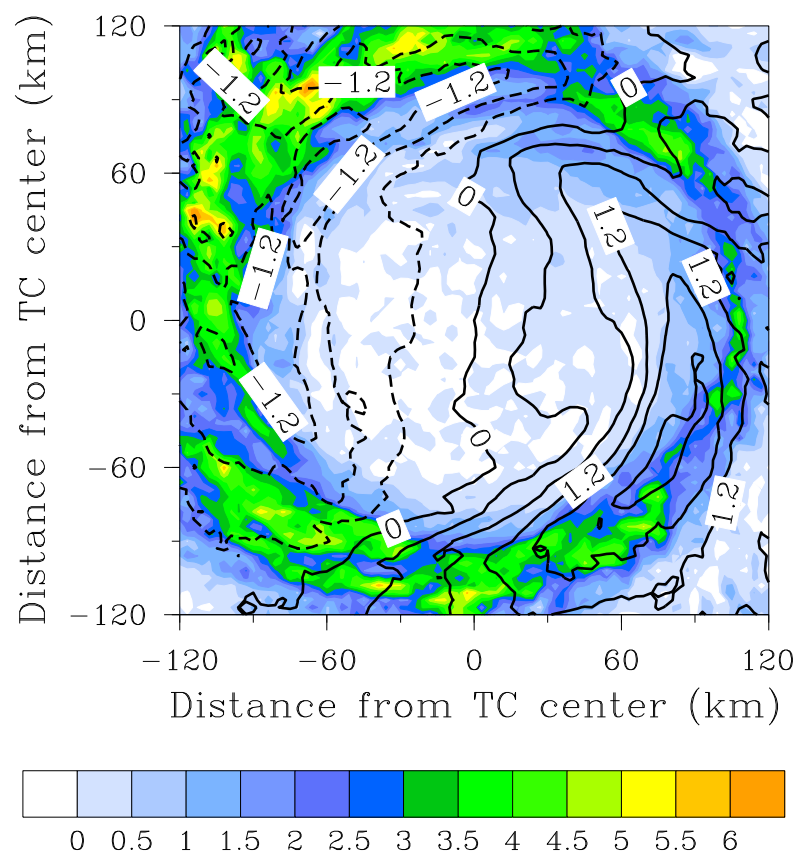

FIG. 14. Plan views of temporally and vertically averaged microphysical diabatic heating rate (shaded; $10^{-3} \mathrm{~K} \mathrm{~s}^{-1}$ ) and temporally averaged asymmetric surface pressure perturbation (contours; $\mathrm{hPa}$; interval: $0.4 \mathrm{hPa}$ ) in COUP between 36 and $48 \mathrm{~h}$. The vertical averaging of diabatic heating rate is taken from 2 to $12 \mathrm{~km}$ in altitude. The asymmetric surface pressure perturbation is obtained by subtracting the symmetric component from the surface pressure.

Figure $15 \mathrm{~b}$ compares the radial distribution of the sum of the Coriolis and centrifugal forces and the radial pressure gradient force in three experiments. Both of these two terms are shown to be reduced by the cold wake and the cold-core eddy, with the role of cold wake being more salient. Although the peak values of these two terms are comparable, the radial pressure gradient force possesses larger radial span than the sum of the Coriolis and centrifugal forces, and therefore leads to inward acceleration of radial wind. A combination of Figs. 15a and 15b suggests that although the stable boundary layer associated with sea surface cooling may lead to smaller surface wind due to diminished turbulent mixing, it does not necessarily cause stronger gradient wind imbalance as concluded by Lee and Chen (2014). This is because the presence of cold wake or cold-core eddy has diminished the diabatic heating and thereby relieved radial pressure gradient, which is shown to be dominant in decreasing the inward acceleration rate. Eventually the radial inflow strength is reduced and the surface inflow angle tends to be decreased by sea surface cooling.

To evaluate the modulating effect of radial inflow on the spinup of TCs, Fig. 16 compares the total differential of tangential wind and the sum of three right-hand-side 
(a) Ihs and rhs terms

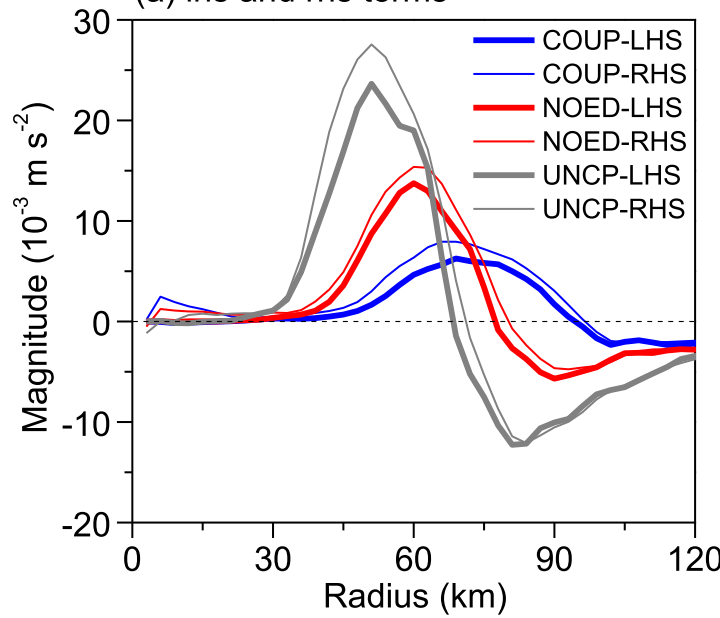

(b) Radial force components

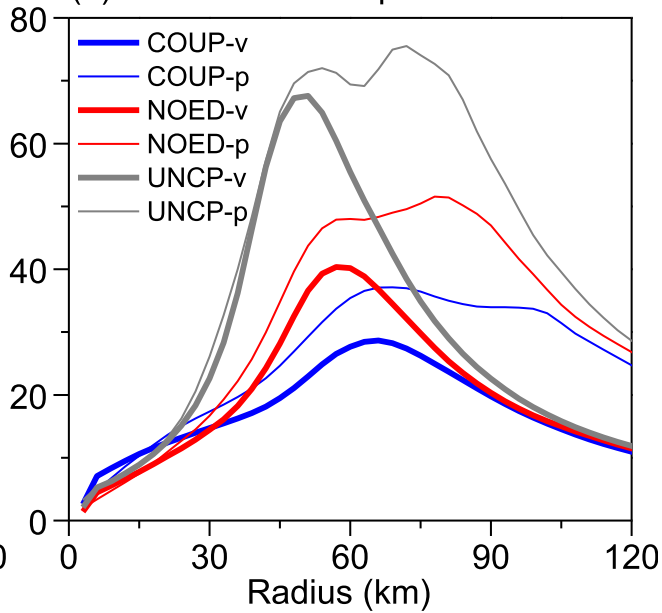

FIG. 15. Radial distribution of azimuthally and temporally averaged (a) $d u / d t$ and the sum of three right-hand-side terms in Eq. (1) $\left(10^{-3} \mathrm{~m} \mathrm{~s}^{-2}\right)$ and (b) $(1 / \rho)(\partial p / \partial r)\left(10^{-3} \mathrm{~m} \mathrm{~s}^{-2}\right)$ and the sum of centrifugal and Coriolis forces $\left(10^{-3} \mathrm{~m} \mathrm{~s}^{-2}\right)$ at the surface between 36 and $48 \mathrm{~h}$.

terms in Eq. (2), and the generalized Coriolis term for three experiments. The generalized Coriolis term denotes inward transport of absolute angular momentum by radial wind and plays a major role in the spinup of primary circulation (Smith et al. 2009). The tangential flow in three experiments accelerates with decreasing radius first when the generalized Coriolis term overwhelms frictional loss, and then decelerates when the total tangential force turns to be negative. The generalized Coriolis term is the smallest in COUP and the largest in UNCP, indicating that the reduced inward transport of absolute angular momentum associated with decreased radial inflow is responsible for the weakened TC intensities by the cold wake and cold-core eddy.

The gradient wind imbalance associated with surface friction is an important characteristic of the TC boundary layer (Smith et al. 2009). Figure 17 displays the time evolution of the extents of gradient wind imbalance at the locations of maximum wind speed and maximum surface wind speed. The simulated storms are characterized by subgradient flow near the surface and supergradient flow near the top of the radial inflow layer. Both the cold-core eddy and cold wake are shown to (a) Ihs and rhs terms

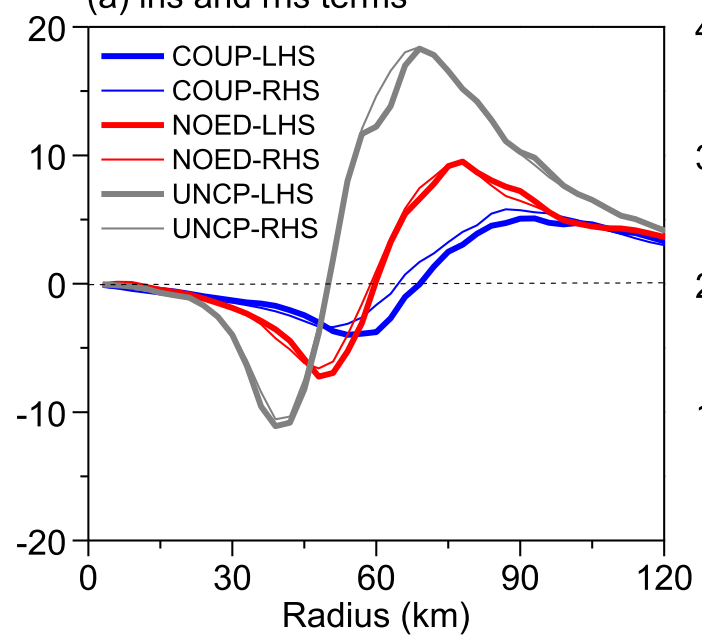

(b) Generalized Coriolis term

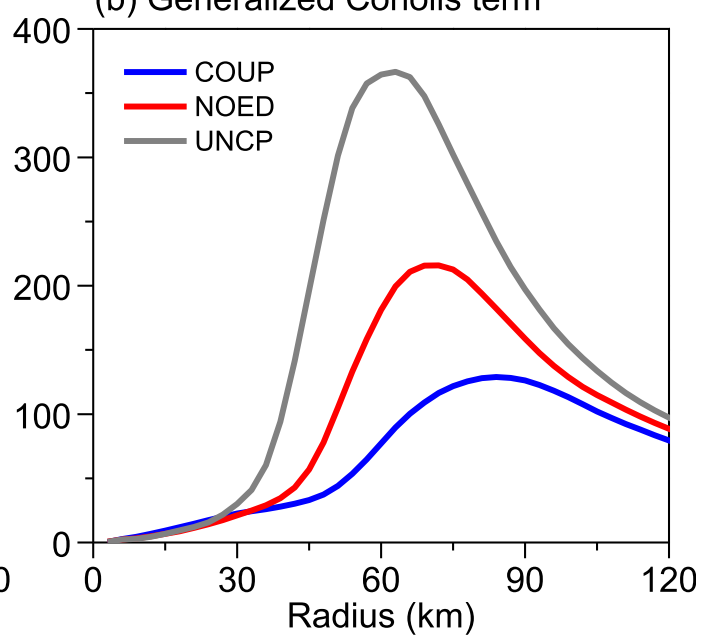

FIG. 16. Radial distribution of azimuthally and temporally averaged (a) $d v / d t$ and the sum of three right-hand-side terms in Eq. (2) $\left(10^{-3} \mathrm{~m} \mathrm{~s}^{-2}\right)$ and (b) generalized Coriolis term $\left(10^{-3} \mathrm{~m} \mathrm{~s}^{-2}\right)$ at the surface between 36 and $48 \mathrm{~h}$. 


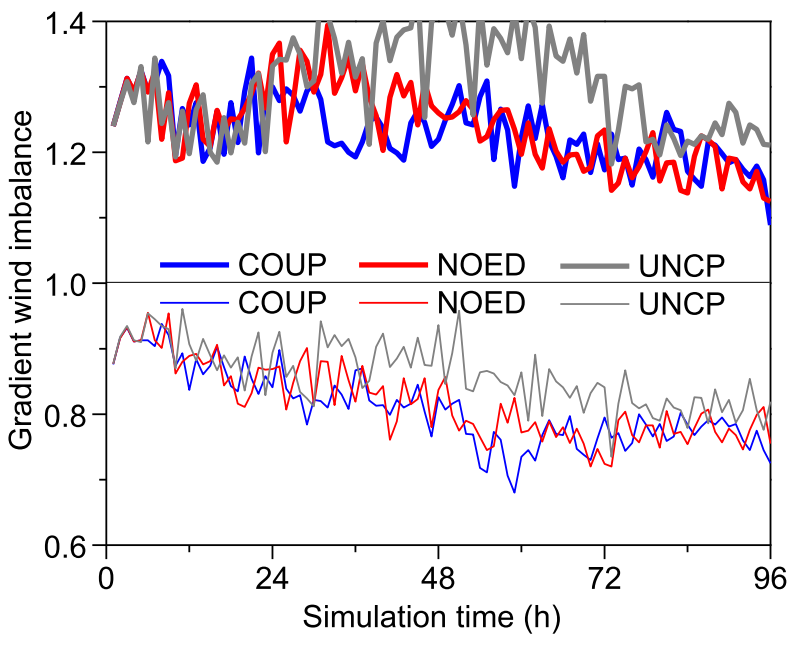

FIG. 17. Time evolution of the extent of gradient wind imbalance at the location of (top) maximum wind speed and (bottom) maximum surface wind.

moderate the extents of gradient wind imbalance. The maximum wind tends to be more supergradient while the maximum surface wind becomes less subgradient due to the presence of cold wake and cold-core eddy, with the role of cold wake being more significant. These can be attributed to the changes in radial pressure gradient force by sea surface cooling and therefore the readjustment of net radial force and frictional stress [Fig. 15; Eq. (1)].

\section{Conclusions}

The effect of cold-core eddy and cold wake on the rapid weakening of Francisco has been investigated in Part I using the atmosphere-ocean coupling model. Based on the model output in Part I, this study extends the work to examine the role of cold-core eddy in modulating the boundary layer structures of Francisco, as well as a comparison with the cold wake effect. The cold-core eddy and the cold wake share some similarities, but discrepancies still exist between them. On one hand, both of them are cold features and contribute negatively to the TC by reducing air-sea enthalpy flux; for this case, they also possess a comparable spatial scale. On the other hand, the cold wake is formed by the TC forcing and gives feedback continuously in the right rear of the TC; the cold-core eddy is a preexisting feature so that it affects the storm shortly when their interaction occurs. Therefore, the eddy feedback depends on the relative location between the TC and the eddy. With Francisco approaching the eddy region, the storm size in terms of the radius of hurricane-force wind decreases, occurring earlier than the eddy-induced enlargement of eye size.
Unlike the inhibited eyewall contraction by the cold wake throughout the simulation, the eddy-induced broadening of the RMW is basically recovered shortly after departure from the eddy region. However, the eddy-induced decrease in storm size does not fully recover at the end of the simulation. The different behaviors between the size and intensity of TCs may be related to different dynamical mechanisms between the outer rainbands and the eyewall. The activities of newly developed rainbands relies closely on the local environment created by preexisting rainbands (Sawada and Iwasaki 2010), and therefore a longer recovery time may be required for the storm size than the intensity after passing over a cold-core eddy.

Both the cold-core eddy and cold wake lead to shallower symmetric HVTH, at comparable rates. Nonetheless, they show distinctly different asymmetric characteristics. Since Francisco translated northwest when interacting with the eddy, the HVTH appears to be shallower in the northwest quadrant of the storm and shifts from the outer region into the eye region. Afterward the decrease in HVTH shifts radially outward as the storm leaves the eddy region gradually. The changes in HVTH correspond well with the relative location between Francisco and the eddy. For the cold wake, however, the HVTH is the shallowest in the right rear of the storm throughout the simulation, and the asymmetric decrease in HVTH is roughly twice that caused by the cold-core eddy. Although the dynamic boundary layer height tends to be shallower due to the presence of cold wake and coldcore eddy, its asymmetric change does not fully comply with the spatial distribution of sea surface cooling.

The cold-core eddy and cold wake not only influence the characteristics of boundary layer height, but also modulate the thermal stability of the boundary layer. The cold-core eddy leads to the formation of stable boundary layer directly above it, which shifts from northwest to southeast following the storm translation. As a result, when the TC center approximately crosses over the eddy center, the inner core of the TC is mostly characterized by stable boundary layer. The cold wakeinduced stable boundary layer is again apparent in the right-rear quadrant of the storm. Together with the formation of stable boundary layer, the sensible heat fluxes at the lowest atmospheric model level are mostly downward over the sea surface cooling region, as previously observed by Black and Holland (1995). In contrast to the important contribution of surface CAPE in the eye that triggered deep convection and therefore rapid intensification of TCs (e.g., Miyamoto and Takemi 2013; Wang and Heng 2016), its role is found to be minor in the rapid weakening of the TC. Nonetheless, the characteristics of surface $\theta_{e}$ have a close relationship with the intensity change of Francisco. The cooling of 
both cold-core eddy and cold wake inhibits the diabatic heating rate associated with convective activities. Due to their different relative locations to the TC, the diabatic heating in the northwest quadrant can be more effectively affected by the cold-core eddy than by the cold wake during the TC-eddy interaction.

The asymmetric characteristics of surface radial wind are found to be sensitive to the presence of cold-core eddy and cold wake, while the surface tangential wind asymmetry is little influenced by sea surface cooling. The asymmetric decrease of surface radial wind caused by the cold-core eddy and cold wake implies changes in surface inflow angle. Results show that the surface inflow angle is decreased especially over the cold-core eddy and cold wake, in a stark contrast to the case of Typhoon Choi-Wan (2009) by Lee and Chen (2014) and the case of Typhoon Megi (2010) by Wu et al. (2016). This at least suggests that the stable boundary layer does not necessarily lead to more inward spiraling airflow, and therefore the positive contribution of stable boundary layer articulated by Lee and Chen (2014) is absent in some TC cases.

A budget analysis based on the radial wind tendency equation indicates that although the Coriolis and centrifugal forces are diminished by sea surface cooling, the decrease in radial pressure gradient caused by coldcore eddy or cold wake is more important in modulating the acceleration rate of surface radial wind. Therefore, the surface inflow strength is decreased, and spiraling surface airflow tends to be more outward due to the presence of cold-core eddy and cold wake. The decrease in radial inflow causes a decrease in generalized Coriolis term, and thereby a more weakened storm. Besides, the maximum wind tends to be more supergradient while the maximum surface wind becomes less subgradient by the sea surface cooling, wherein the role of cold wake is more significant than that of coldcore eddy.

Finally, the conclusions drawn from above are based on a single case in rapid weakening period. More efforts on various TC cases or statistical analysis may still be expected to generalize the results reported herein. More aspects of TC-eddy interaction, such as in the context of sea spray processes and sea waves, are still not well understood and will be explored in our future work.

Acknowledgments. The authors appreciate the important comments from two anonymous reviewers for improving the manuscript. The authors also thank Prof. Black for recommending several beneficial references. This work is supported by the National Natural Science Foundation of China with Grants 41875062 and 41675058.

\section{REFERENCES}

Anthes, R. A., and S. W. Chang, 1978: Response of the hurricane boundary layer to changes of sea surface temperature in a numerical model. J. Atmos. Sci., 35, 1240-1255, https://doi.org/ 10.1175/1520-0469(1978)035<1240:ROTHBL>2.0.CO;2.

Barnes, G. M., and M. D. Powell, 1995: Evolution of the inflow boundary layer of Hurricane Gilbert (1988). Mon. Wea. Rev., 123, 2348-2368, https://doi.org/10.1175/1520-0493(1995) $123<2348$ :EOTIBL $>2.0$.CO; 2 .

- , and P. Fuentes, 2010: Eye excess energy and the rapid intensification of Hurricane Lili (2002). Mon. Wea. Rev., 138, 1446-1458, https://doi.org/10.1175/2009MWR3145.1.

Bhalachandran, S., R. Nadimpalli, K. K. Osuri, F. D. Marks, S. Gopalakrishnan, S. Subramanian, U. C. Mohanty, and D. Niyogi, 2019: On the processes in influencing rapid intensity changes of tropical cyclones over the Bay of Bengal. Sci. Rep., 9, 3382, https://doi.org/10.1038/s41598-019-40332-z.

Black, P. G., and G. J. Holland, 1995: The boundary layer in Tropical Cyclone Kerry (1979). Mon. Wea. Rev., 123, 2007-2028, https:// doi.org/10.1175/1520-0493(1995)123<2007:TBLOTC>2.0.CO;2.

Bryan, G. H., and R. Rotunno, 2009: The maximum intensity of tropical cyclones in axisymmetry numerical model simulations. Mon. Wea. Rev., 137, 1770-1789, https://doi.org/10.1175/ 2008MWR2709.1.

Chan, K., and J. Chan, 2015: Impacts of vortex intensity and outer winds on tropical cyclone size. Quart. J. Roy. Meteor. Soc., 141, 525-537, https://doi.org/10.1002/qj.2374.

Chen, S., T. J. Campbell, H. Jin, S. Gabersek, R. M. Hodur, and P. Martin, 2010: Effect of two-way air-sea coupling in high and low wind speed regimes. Mon. Wea. Rev., 138, 3579-3602, https://doi.org/10.1175/2009MWR3119.1.

Emanuel, K. A., 1986: An air-sea interaction theory for tropical cyclones. Part I: Steady-state maintenance. J. Atmos. Sci., 43, 585-605, https://doi.org/10.1175/1520-0469(1986)043<0585: AASITF $>2.0 . \mathrm{CO} ; 2$.

Fang, J., and F. Zhang, 2011: Evolution of multiscale vortices in the development of Hurricane Dolly (2008). J. Atmos. Sci., 68 , 103-122, https://doi.org/10.1175/2010JAS3522.1.

Gopalakrishnan, S. G., F. D. Marks Jr., X. Zhang, J.-W. Bao, K.-S. Yeh, and R. Atlas, 2011: The experimental HWRF system: A study on the influence of horizontal resolution on the structure and intensity changes in tropical cyclones using an idealized framework. Mon. Wea. Rev., 139, 1762-1784, https://doi.org/ 10.1175/2010MWR3535.1.

-, J. A. Zhang, X. Zhang, J.-W. Bao, and V. Tallapragad, 2013: A study of the impacts of vertical diffusion on the structure and intensity of the tropical cyclones using the highresolution HWRF system. J. Atmos. Sci., 70, 524-541, https:// doi.org/10.1175/JAS-D-11-0340.1.

Hong, S. Y., Y. Noh, and J. Dudhia, 2006: A new vertical diffusion package with an explicit treatment of entrainment processes. Mon. Wea. Rev., 134, 2318-2341, https://doi.org/10.1175/MWR3199.1.

Hong, X., S. W. Chang, S. Raman, L. K. Shay, and R. Hodur, 2000: The interaction between Hurricane Opal (1995) and a warm core ring in the Gulf of Mexico. Mon. Wea. Rev., 128, 1347-1365, https:// doi.org/10.1175/1520-0493(2000)128<1347:TIBHOA > 2.0.CO;2.

Kepert, J. D., 2001: The dynamics of boundary layer jets within the tropical cyclone core. Part I: Linear theory. J. Atmos. Sci., 58, 2469-2484, https://doi.org/10.1175/1520-0469(2001)058<2469: TDOBLJ $>2.0 . \mathrm{CO} ; 2$.

, 2010: Tropical cyclone structure and dynamics. Global Perspectives on Tropical Cyclones: From Science to Mitigation, 
J. C. L. Chan and J. D. Kepert, Eds., World Scientific Series on Asia-Pacific Weather and Climate, Vol. 4, World Scientific, 3-53.

— - J. Schwendike, and H. Ramsay, 2016: Why is the tropical cyclone boundary layer not "well mixed"? J. Atmos. Sci., 73, 957-973, https://doi.org/10.1175/JAS-D-15-0216.1.

Kieu, C. Q., 2012: An investigation into the contraction of the hurricane radius of maximum wind. Meteor. Atmos. Phys., 115, 47-56, https://doi.org/10.1007/s00703-011-0171-7.

Lee, C.-Y., and S. S. Chen, 2012: Symmetric and asymmetric structures of hurricane boundary layer in coupled atmospherewave-ocean models and observations. J. Atmos. Sci., 69 , 3576-3594, https://doi.org/10.1175/JAS-D-12-046.1.

— tropical cyclone structure in a coupled atmosphere-ocean model. Mon. Wea. Rev., 142, 1927-1944, https://doi.org/10.1175/ MWR-D-13-00122.1.

Lin, I.-I., C.-C. Wu, K. A. Emanuel, I.-H. Lee, C.-R. Wu, and I.-F. Pum, 2005: The interaction of Supertyphoon Maemi with a warm ocean eddy. Mon. Wea. Rev., 133, 2635-2649, https:// doi.org/10.1175/MWR3005.1.

Ma, Z., 2020: A study of the interaction between Typhoon Francisco (2013) and a cold-core eddy. Part I: Rapid weakening. J. Atmos. Sci., 77, 355-377, https://doi.org/10.1175/JAS-D-18-0378.1.

— J. Fei, X. Huang, and X. Cheng, 2013a: The effects of ocean feedback on tropical cyclone energetics under idealized airsea interaction conditions. J. Geophys. Res. Atmos., 118, 9778 9788, https://doi.org/10.1002/JGRD.50780.

,,-- L. Liu, X. Huang, and X. Cheng, 2013b: Effects of the cold core eddy on tropical cyclone intensity and structure under idealized air-sea interaction conditions. Mon. Wea. Rev., 141, 1285-1303, https://doi.org/10.1175/MWR-D-1200123.1.

,$- \ldots$ X. Huang, and X. Cheng, 2015: Contributions of surface sensible heat fluxes to tropical cyclone. Part I: Evolution of tropical cyclone intensity and structure. J. Atmos. Sci., 72, 120140, https://doi.org/10.1175/JAS-D-14-0199.1.

Malkus, J. S., and H. Riehl, 1960: On the dynamics and energy transformations in steady-state hurricane. Tellus, 12, 1-20, https://doi.org/10.3402/tellusa.v12i1.9351.

Miyamoto, Y., and T. Takemi, 2013: A transition mechanism for the spontaneous axisymmetric intensification of tropical cyclones. J. Atmos. Sci., 70, 112-129, https://doi.org/10.1175/ JAS-D-11-0285.1.

Molinari, J., D. M. Romps, D. Vollaro, and L. Nguyen, 2012: CAPE in tropical cyclones. J. Atmos. Sci., 69, 2452-2463, https:// doi.org/10.1175/JAS-D-11-0254.1.

Montgomery, M., M. E. Nicholls, T. A. Cram, and A. B. Saunders, 2006: A vortical hot tower route to tropical cyclogenesis. J. Atmos. Sci., 63, 355-386, https://doi.org/10.1175/JAS3604.1.

Pendergrass, A. G., and H. E. Willoughby, 2009: Diabatically induced secondary flows in tropical cyclones. Part I: Quasi-steady forcing. Mon. Wea. Rev., 137, 805-821, https://doi.org/10.1175/ 2008MWR2657.1.

Powell, M. D., 1990: Boundary layer structure and dynamics in outer hurricane rainbands. Part II: Downdraft modification and mixed layer recovery. Mon. Wea. Rev., 118, 918-938, https://doi.org/10.1175/1520-0493(1990)118<0918:BLSADI > 2.0.CO;2.

Rogers, R., 2010: Convective-scale structure and evolution during a high-resolution simulation of tropical cyclone rapid intensification. J. Atmos. Sci., 67, 44-70, https://doi.org/ 10.1175/2009JAS3122.1.

, P. Reasor, and S. Lorsolo, 2013: Airborne Doppler observations of the inner-core structural differences between intensifying and steady-state tropical cyclones. Mon. Wea. Rev., 141, 2970-2991, https://doi.org/10.1175/MWR-D-12-00357.1.

Sawada, M., and T. Iwasaki, 2010: Impacts of evaporation from raindrops on tropical cyclones. Part II: Features of rainbands and asymmetric structure. J. Atmos. Sci., 67, 84-96, https:// doi.org/10.1175/2009JAS3195.1.

Shapiro, L. J., and H. E. Willoughby, 1982: The response of balanced hurricanes to local sources of heat and momentum. J. Atmos. Sci., 39, 378-394, https://doi.org/10.1175/1520-0469(1982) 039<0378:TROBHT $>2.0 . \mathrm{CO} ; 2$.

Shay, L. K., G. J. Goni, and P. G. Black, 2000: Effects of a warm oceanic feature on Hurricane Opal. Mon. Wea. Rev., 128, 1366-1383, https://doi.org/10.1175/1520-0493(2000)128<1366: EOAWOF $>2.0 . \mathrm{CO} ; 2$.

Smith, R. K., M. T. Montgomery, and V. S. Nguyen, 2009: Tropical cyclone spin-up revisited. Quart. J. Roy. Meteor. Soc., 135, 1321-1335, https://doi.org/10.1002/qj.428.

Stern, D. P., J. L. Vigh, D. S. Nolan, and F. Zhang, 2015: Revisiting the relationship between eyewall contraction and intensification. J. Atmos. Sci., 72, 1283-1306, https://doi.org/10.1175/ JAS-D-14-0261.1.

Stull, R. B., 1988: An Introduction to Boundary Layer Meteorology. Kluwer Academic, 666 pp.

Vigh, J. L., and W. H. Schubert, 2009: Rapid development of the tropical cyclone warm core. J. Atmos. Sci., 66, 3335-3350, https://doi.org/10.1175/2009JAS3092.1.

Walker, N. D., R. R. Leben, C. T. Pilley, M. Shannon, D. C. Herndon, I.-F. Pun, I.-I. Lin, and C. L. Gentemann, 2014: Slow translation speed causes rapid collapse of northeast Pacific Hurricane Kenneth over cold core eddy. Geophys. Res. Lett., 41, 7595-7601, https://doi.org/10.1002/2014GL061584.

Wang, Y., and J. Heng, 2016: Contribution of eye excess energy to the intensification rate of tropical cyclones: A numerical study. J. Adv. Model. Earth Syst., 8, 1953-1968, https://doi.org/10.1002/ 2016MS000709.

Willoughby, H. E., J. A. Clos, and M. G. Shoreibah, 1982: Concentric eye walls, secondary wind maxima, and the evolution of the hurricane vortex. J. Atmos. Sci., 39, 395-411, https://doi.org/ 10.1175/1520-0469(1982)039<0395:CEWSWM > 2.0.CO;2.

Wu, C.-C., W.-T. Tu, I.-F. Pun, I.-I. Lin, and M. S. Peng, 2016: Tropical cyclone-ocean interaction in Typhoon Megi (2010)-A synergy study based on ITOP observations and atmosphereocean coupled model simulations. J. Geophys. Res. Atmos., 121, 153-167, https://doi.org/10.1002/2015JD024198.

Zhang, J. A., R. F. Rogers, D. S. Nolan, and F. D. Marks Jr., 2011: On the characteristic height scales of the hurricane boundary layer. Mon. Wea. Rev., 139, 2523-2535, https://doi.org/10.1175/ MWR-D-10-05017.1.

, M. T. Montgomery, F. D. Marks Jr., and R. K. Smith, 2014: Comments on "Symmetric and asymmetric structures of hurricane boundary in coupled atmosphere-wave-ocean models and observations."' J. Atmos. Sci., 71, 2782-2785, https://doi.org/ 10.1175/JAS-D-13-0207.1.

Zhu, H., U. Wolfgang, and S. K. Roger, 2004: Ocean effects on tropical cyclone intensification and inner-core asymmetries. J. Atmos. Sci., 61, 1245-1258, https://doi.org/10.1175/15200469(2004)061<1245:OEOTCI $>2.0$. CO 2 . 